\title{
Alpine meadow degradation depresses soil nitrogen fixation by regulating plant functional groups and diazotrophic community composition
}

\author{
Lu Zhang \\ Chinese Academy of Sciences and Ministry of Water Resources \\ Xiangtao Wang \\ Xizang Agriculture and Animal Husbandry College \\ Jie Wang \\ Northwest A\&F University \\ Lirong Liao \\ Chinese Academy of Sciences and Ministry of Water Resources \\ Shilong Lei \\ Northwest A\&F University \\ Guobin Liu \\ Chinese Academy of Sciences and Ministry of Water Resources \\ Chao Zhang ( $\nabla$ zhangchao1985@nwafu.edu.cn) \\ Northwest Agriculture and Forestry University https://orcid.org/0000-0003-2647-2922
}

\section{Research Article}

Keywords: Biological nitrogen fixation, alpine meadow, nifH gene, diazotrophic community

Posted Date: July 28th, 2021

DOl: https://doi.org/10.21203/rs.3.rs-744121/v1

License: (c) (i) This work is licensed under a Creative Commons Attribution 4.0 International License. Read Full License

Version of Record: A version of this preprint was published at Plant and Soil on January 4th, 2022. See the published version at https://doi.org/10.1007/s11104-021-05287-z. 


\section{Abstract}

\section{Aims}

Biological nitrogen fixation (BNF), a function performed by diazotrophic microbes, plays an essential role in nitrogen (N) bioavailability in terrestrial ecosystems. However, little is known about the effects of degradation on soil BNF and diazotrophic communities in alpine meadow.

\section{Methods}

We investigated the changes in soil BNF and their potential drivers in alpine meadows along a degradation gradient on the Tibetan Plateau (non-degraded, lightly degraded, moderately degraded, and severely degraded meadows) using real-time quantitative PCR and amplicon sequencing.

\section{Results}

Soil BNF rates decreased significantly along the meadow degradation gradient with a range of 17.34-79.84 $\mathrm{nmol} \mathrm{C}_{2} \mathrm{H}_{4} \mathrm{~g}^{-}$

${ }^{1}$ dry soil $\mathrm{d}^{-1}$ across all sites. The highest BNF was observed in the non-degraded meadow and was 1.5-4.6-fold higher than that in degraded meadows. Meadow degradation significantly reduced the gene abundance of nifH and the Shannon and Chao1 diversity indices of diazotrophs, accompanied by a decrease in plant biomass, soil moisture, and nutrient content (C, N component). Soil BNF potential was closely correlated with plant biomass, soil nutrient content, and diazotrophic abundance (including Nostoc, Scytonema, Rhodopseudomonas, Rhizobiales, and Proteobacteria). The community composition of diazotrophs differed markedly among sites with different levels of degradation, and both autotrophic (Cyanobacteria) and heterotrophic (Proteobacteria) diazotrophs contributed simultaneously to the BNF. The plant functional groups, especially the sedges family, were the primary drivers for soil BNF rates via mediating soil moisture, nutrient level (dissolved organic $\mathrm{C}$ and $\mathrm{N}$ ), nifH gene abundance, and diazotrophic community composition.

\section{Conclusions}

Our results reveal the underlying mechanism of changes in soil BNF during alpine meadow degradation, emphasize the importance of plant functional groups in shaping the diazotrophic community and BNF potential, and provide insights for the restoration of degraded meadow ecosystems.

\section{Introduction}

Alpine meadows are widely distributed in the Tibetan Plateau-covering a total area of $700000 \mathrm{~km}^{2}$-and play numerous roles in soil and water conservation, carbon (C) sequestration, climate regulation, and habitat conservation (Che et al. 2019; Wang et al. 2020b). However, in the past 30 years, large areas of alpine meadows have experienced severe degradation due to severe climate change and anthropogenic activities (such as overgrazing and tramping), which poses an increasing threat to the resistance and sustainability of alpine ecosystems (Fu et al. 2012; Luo et al. 2018; Wen et al. 2010). Meadow degradation is usually characterized by the destruction of plant communities, soil drought, soil nutrient deterioration, and even desertification, which are serious environmental problems (Urak et al. 2017; Yang et al. 2013). In addition, the low soil nitrogen $(\mathrm{N})$ content in alpine meadows is a critical factor limiting the restoration and development of ecosystems (Colin et al. 2019; Zhang et al. 2020b). Meadow degradation can accelerate the loss of soil N, further increasing $\mathrm{N}$ limitation in alpine ecosystems (Kou et al. 2019). Although the dynamics of $\mathrm{N}$ in the pedosphere have been 
widely studied, our understanding of how meadow degradation affects $\mathrm{N}$ input is still largely lacking, which may hinder the modeling and prediction of $\mathrm{N}$ dynamics in alpine ecosystems.

Biological nitrogen fixation (BNF) is a microbial process that reduces $\mathrm{N}_{2}$ gas to bioavailable ammonium $\left(\mathrm{NH}_{4}{ }^{+}-\mathrm{N}\right)$ via catalysis by nitrogenase in the biosphere, which is a dominant input of $\mathrm{N}$ in meadow ecosystems with extensive $\mathrm{N}$ limitation (Vicente and Dean 2017; Wang et al. 2017). BNF occurs via two ways: symbiotic and free-living $\mathrm{N}_{2}$ fixation (Che et al. 2018; Reed et al. 2011; Wang et al. 2021c), in which symbiotic $\mathrm{N}_{2}$ fixation mainly occurs in the root or stem nodules of legume plants, and free-living $\mathrm{N}_{2}$ fixation, which occurs independently of legume-microbe mutualism and may be sensitive to environmental change (Lindstroem and Mousavi 2020; Smercina et al. 2019). In addition, although the rate of free-living BNF is lower than that of symbiotic fixation, in N-limited ecosystems where most plants lack $\mathrm{N}_{2}$-fixation ability, free-living $\mathrm{N}_{2}$ fixation may be one of the most important pathways for soil $\mathrm{N}$ accumulation (Reed et al. 2011; Wang et al. 2021c). Hence, understanding the abundance, diversity, and composition of these communities is imperative to elucidating their ecological functions in alpine ecosystems.

Due to the highly conserved nature of the $\mathrm{nifH}$ gene that encodes nitrogenase, it has been widely used as a molecular marker to study the abundance and community composition of $\mathrm{N}_{2}$-fixing bacteria in soil (Fan et al. 2019; Levy-Booth et al. 2014; Mårtensson et al. 2009). However, although the process of BNF is well documented, how nifH genes and diazotrophic communities respond to alpine meadow degradation is still not understood. The absolute abundance of the nifH gene has a significant positive correlation with BNF and can be used to indicate the strength of BNF (Che et al. 2019; Levy-Booth et al. 2014). However, the research of Kumar et al. (2017) showed that the nifH gene is not a good criterion for judging the potential for BNF. The function of $\mathrm{N}_{2}$-fixation in soil is determined based on the diazotrophic community composed of multiple distinct $\mathrm{N}_{2}$-fixation taxa (Che et al. 2017; Dai et al. 2021; Wang et al. 2020a). Moreover, functional redundancy is widespread in microbial systems during exerting metabolic functions (Louca et al. 2018). Therefore, to better explore the driving mechanism of soil BNF in alpine ecosystems, the community composition and diversity of diazotrophs should be considered. Moreover, the biotic and abiotic pathways that regulate BNF are also unclear, especially in alpine meadow ecosystems.

Several studies have reported the effects of meadow degradation or restoration on BNF (Wang et al. 2017; Wen et al. 2010; Zhang et al. 2020b). Changes in plant community structure caused by meadow degradation are one of the important reasons for changes in soil BNF (Zhang et al. 2020b). Plant roots can regulate the rhizosphere micro-ecosystem and affect the metabolic process of diazotrophs by releasing root exudates, thus directly affecting the activity of diazotrophs (PerezMontano et al. 2014; Wang et al. 2020b). Revegetation can also promote the formation of diverse diazotrophic communities by causing an increase in the abundance and diversity of microbes, and thus regulating the BNF potential (Huang et al. 2011; Li et al. 2021). Indirectly, the decomposition of plant residues can provide organic matter and other effective substances, that change soil physicochemical properties (Yang et al. 2021). The activity, abundance, diversity, and composition of $\mathrm{N}_{2}$-fixing bacteria are highly sensitive to variations in soil physicochemical properties, including soil moisture, pH, temperature, nutrients, and carbon (C), N, and phosphorus (P) availability (Fan et al. 2019; Li et al. 2021; Reed et al. 2011). In addition, plant functional groups (PFGs) are important factors that connect plant community characteristics and ecosystem processes, and variation in PFGs can generate feedback regulation on soil-microbe interactions (Wang et al. 2021a; Wu et al. 2019). Luo et al. (2018) confirmed that the community structure of PFG can be used to indicate the degradation process of alpine grasslands on the Tibetan Plateau. Species in a PFG have similar strategies in response to environmental disturbance; therefore, PFGs are crucial for the study of ecosystem functions, especially BNF (Zhang et al. 2020b). Thus, exploring the linkages between PFGs, soil, and diazotrophs could improve our understanding of the regulation of soil BNF with meadow degradation.

In this study, we aimed to assess the impacts of meadow degradation on the diazotrophic community structure and soil BNF potential on the Tibetan Plateau. Here, we sequenced the nifH gene and quantified its abundance to assess the soil 
diazotrophic communities of alpine meadows with different degradation levels in the Tibetan Plateau (non-degraded, lightly degraded, moderately degraded, and severely degraded meadows). We hypothesized that: (1) meadow degradation significantly reduces soil BNF rate and changes plant and diazotrophic community structure; and (2) plant communities, especially PFGs, are the main influencing factors of soil BNF rate and diazotrophic community structure. To verity the above-mentioned hypotheses, the following topics were addressed: (1) the main variation in soil physicochemical properties and plant community caused by meadow degradation; (2) how meadow degradation affects the soil BNF rate and abundance, composition, and diversity of the diazotrophic community; and (3) the possible drivers of soil BNF rate and diazotrophic community.

\section{Materials And Methods}

\subsection{Description of study site}

The study was conducted at the grassland station $\left(91^{\circ} 05^{\prime} \mathrm{E}, 30^{\circ} 51^{\prime} \mathrm{N}, 4333 \mathrm{~m} \mathrm{ASL}\right)$ of the Alpine Meadow Nature Reserve of Damxung County in the mid-south portion of the Tibetan Plateau. This area is characterized by a typical semi-arid alpine continental climate. The annual mean precipitation and air temperature in this area are $477 \mathrm{~mm}$ and $1.3{ }^{\circ} \mathrm{C}$, respectively (Wang et al. 2021b). Approximately $85 \%$ of the precipitation mainly occurs from July to August during the summer monsoon season. The lowest temperature is $-10.4{ }^{\circ} \mathrm{C}$ in January, and the highest temperature is $10.7^{\circ} \mathrm{C}$ in July. There are approximately 62 frost-free days in a year, with an annual accumulated temperature $\left(\geq 0{ }^{\circ} \mathrm{C}\right)$ of $1800^{\circ} \mathrm{C}$. In the study area, the soil is mainly classified as mountain meadow soil (Cambisol, FAO/UNESCO classification), and had a sandy loam texture. The meadow is dominated by Kobresia pygmaea, Stipa capillacea, and Carex montis-everestii (Fu et al. 2012).

\subsection{Experimental design and sample collection}

Four meadows were established along a degradation gradient (non-degraded, ND; lightly degraded, LD; moderately degraded, MD; and severely degraded, SD meadows) by the Chinese Academy of Sciences in this nature reserve, with an

area of $15-20 \mathrm{hm}^{2}$. Based on the results of vegetation survey (Tables S2 and S3), a meadow index was used to evaluate the degree of meadow degradation calculating as described by Wen et al. (2010). Five $50 \times 50 \mathrm{~m}$ plots were set up randomly with similar terrain conditions in each meadow, with a distance of more than 80-100 m between each plot. In total, 20 plots were established in a randomized complete block design.

Five quadrats $(1 \mathrm{~m} \times 1 \mathrm{~m})$ were set in each plot to conduct a vegetation survey and soil sampling in August 2019. Soil subsamples were randomly collected at a depth of $15 \mathrm{~cm}$ per quadrat along an S-shaped pattern (nine cores per quadrat) by a sterile soil auger with a diameter of $10 \mathrm{~cm}$. A composite sample was formed by evenly mixing all sub-samples from five quadrats. After garbage, stone, and debris were removed, each soil sample was filtered through a 2-mm sieve and divided into two subsamples. One subsample was stored at $-80^{\circ} \mathrm{C}$ for DNA extraction, and another was air-dried to analyze its physicochemical properties. After soil sampling, the plants in each quadrat were dug up and sorted into functional groups (including sedges, grasses, forbs, and legumes) as described by Wu et al. (2019). The above- and belowground parts of each plant species were carefully separated, cleaned, dried at $70^{\circ} \mathrm{C}$ for $48 \mathrm{~h}$, and weighed to obtain the above- and belowground biomass. The plant Shannon diversity index was calculated according to the method described by Zhang et al. (2016).

\subsection{Soil physicochemical analysis}

Soil moisture (SM) was determined by oven-drying fresh soil at $105^{\circ} \mathrm{C}$ for $24 \mathrm{~h}$. As described by Zhang et al. (2018), the concentrations of $\mathrm{NH}_{4}{ }^{+}-\mathrm{N}$ and $\mathrm{NO}_{3}{ }^{-}-\mathrm{N}$ were extracted with $2 \mathrm{M} \mathrm{KCl}(1: 10 \mathrm{w} / \mathrm{v})$ and subsequently analyzed using a segmented-flow autoanalyzer system. The Kjeldahl digestion method was used to determine soil total nitrogen (TN). After extraction with distilled water, dissolved organic nitrogen (DON) and dissolved organic carbon (DOC) were analyzed using 
a TOC/TN analyzer. Soil organic carbon (SOC) was determined using potassium dichromate $\left(\mathrm{K}_{2} \mathrm{Cr}_{2} \mathrm{O}_{7}\right)$ oxidation. Total phosphorus (TP) was determined by melt-molybdenum, antimony, and scandium colorimetry. Soil available potassium (AK) was extracted with $1.0 \mathrm{M} \mathrm{CH}_{3} \mathrm{COONH}_{4}$ at a pH of 7.0 and determined using a flame photometer. Soil available phosphorus (AP) was extracted with $0.5 \mathrm{M} \mathrm{NaHCO}_{3}$ at a pH of 8.5 and measured with a UV spectrophotometer by the method of molybdate ascorbic acid. Soil pH was measured with a pH meter (Spectrum Technologies Inc., Bridgend, UK) using a soil-water ratio of 1:2.5 (w/v) prepared with air-dried soil.

\subsection{Potential biological $\mathrm{N}_{2}$ fixation rate}

The potential BNF rate was measured by the acetylene $\left(\mathrm{C}_{2} \mathrm{H}_{2}\right)$ reduction assay (Patra et al. 2006). In brief, fresh soil (10 g on dried basis) from each sample was transferred into a sterile bottle $(100 \mathrm{ml})$. In each bottle, $10 \%$ of the headspace air was replaced by $\mathrm{C}_{2} \mathrm{H}_{2}(99.99 \%)$ with a gas syringe. The flasks were incubated for 7 days at $25^{\circ} \mathrm{C}$. After incubations, a mixed gas sample $(20 \mathrm{ml})$ was extracted from the headspace of each bottle and placed into a $12 \mathrm{ml}$ pre-evacuated airtight vial using a gas syringe. The concentration of ethylene $\left(\mathrm{C}_{2} \mathrm{H}_{4}\right)$ in the gas samples was measured using a gas chromatograph. Each sample was prepared and measured in triplicates, and the average value for each sample was calculated. The potential BNF rates were expressed as the rate of $\mathrm{C}_{2} \mathrm{H}_{4}$ production rate $\left(n m o l \mathrm{C}_{2} \mathrm{H}_{4} \mathrm{~g}^{-1} \mathrm{dry}\right.$ soil d $\left.\mathrm{d}^{-1}\right)$. We also conducted blank experiments (no soil) to assess the background $\mathrm{C}_{2} \mathrm{H}_{4}$ concentration of $\mathrm{C}_{2} \mathrm{H}_{2}$ and found that in these samples, the $\mathrm{C}_{2} \mathrm{H}_{4}$ concentrations were lower than the detection limits of the gas chromatograph.

\subsection{Microbial analysis}

\subsubsection{Microbial DNA extraction and quantitative PCR analysis}

The DNA was extracted from $0.5 \mathrm{~g}$ of soil using the FastDNA® SPIN Kit, (MP Biomedicals, Cleveland, USA) following the manufacturer's instructions. The quality and concentration of DNA were checked using a NanoDrop 2000 spectrometer (Thermo Scientific, Wilmington, DE, USA). The gene abundance of nifH were determined by an ABI Prism 7500 Real-Time quantitative PCR (qPCR) system (Applied Biosystems, Foster City, CA, USA) using primer pairs nifHF (5'-

AAAGGYGGWATCGGYAARTCCACCAC-3') and nifHR (5'-TTGTTSGCSGCRTACATSGCCATCAT-3') (Rosch and Bothe 2005). The $20 \mu \mathrm{L}$ qPCR reaction mixture consisted of $10 \mu \mathrm{L}$ SYBR® Premix Ex Taq ${ }^{\text {TM }}$ (Takara Bio USA), $1 \mu \mathrm{L}$ DNA template, $0.8 \mu \mathrm{L}$ each of the forward and reverse primers, and $7.4 \mu \mathrm{L}$ double-distilled water $\left(\mathrm{ddH}_{2} \mathrm{O}\right)$. The qPCR program run as follows: denaturation at $98^{\circ} \mathrm{C}$ for $5 \mathrm{~min}$, then 35 cycles of denaturation at $95^{\circ} \mathrm{C}$ for $30 \mathrm{~s}$, annealing at $58^{\circ} \mathrm{C}$ for $30 \mathrm{~s}$, and extension at $72^{\circ} \mathrm{C}$ for $60 \mathrm{~s}$. The qPCR was carried out in triplicate for each sample and the negative control (without DNA template) for each experiment was run simultaneously. Standard curves were obtained with ten-fold serially diluted plasmids of the nifH gene. The nifH gene was quantified based on the average slope value obtained from the standard curves.

\subsubsection{Amplicon sequencing and phylogenetic classification}

After DNA extraction, the community composition and diversity of diazotrophs were analyzed using Illumina MiSeq sequencing technology (Illumina Inc.) and the primer sets nifHF/nifH R (Rosch and Bothe 2005). The $20 \mu \mathrm{L}$ PCR reaction mixture consisted of containing $4 \mu \mathrm{L} 5 \times$ FastPfu buffer, $2 \mu \mathrm{L}$ dNTPs $(2.5 \mathrm{mM}), 0.8 \mu \mathrm{L}$ of each primer $(5 \mu \mathrm{M}), 0.4 \mu \mathrm{L}$ FastPfu polymerase, $0.2 \mu \mathrm{L}$ bovine serum albumin, $10 \mathrm{ng}$ template DNA, and $\mathrm{dd}_{2} \mathrm{O}$. The PCR reactions were performed using TransGen AP221-02 TransStart Fastpfu DNA Polymerase (Transgen Biotech Co., Ltd.) on an ABI GeneAmp ${ }^{8} 9700$ PCR System (Applied Biosystems). The PCR cycling program was as follows: initial denaturation at $95^{\circ} \mathrm{C}$ for $3 \mathrm{~min}$, followed by 35 cycles at $95^{\circ} \mathrm{C}$ for $30 \mathrm{~s}$, annealing at $55^{\circ} \mathrm{C}$ for $30 \mathrm{~s}, 72^{\circ} \mathrm{C}$ for $45 \mathrm{~s}$, and a final extension at $72^{\circ} \mathrm{C}$ for $10 \mathrm{~min}$. PCR amplicons were purified by agarose gel electrophoresis (1.5\% gel agar, $90 \mathrm{~V}, 45 \mathrm{~min}$ ). PCR amplification was conducted in triplicate for each sample, pooled as a PCR product, and then sequenced using the Illumina MiSeq PE300 platform (Majorbio Company in Shanghai, China). 
The QIIME-1.9.1 pipeline (https://docs.qiime2.org/2021.4/citation/) was used to analyze the raw nucleotide sequences of nifH. Quality-controlled sequences were obtained after filtering the adaptors, barcodes, and low-quality sequences (quality score $<20$, containing ambiguous nucleotides or not matching the primer). Operational taxonomic units (OTUs) were clustered using UCLUST with a 97\% similarity cut-off (Edgar 2010). Taxonomic identity was determined using the RDP classifier (Version 2.2, http://rdp.cme.msu.edu/) in the FunGene (Version 9.6, http://fungene.cme.msu.edu/) database (Fish et al. 2013). The Shannon diversity and Chao1 richness indices were calculated by Mothur software v. 1.30.2 (https://mothur.org/CITATION/).

\subsection{Statistical analysis}

One-way analysis of variance and Duncan's multiple tests were used to assess the significant differences in plant and soil properties, soil BNF rate, and diazotrophs among the four meadows $(P<0.05)$. Nonmetric multidimensional scaling (NMDS) ordinations and permutational multivariate analysis of variance ANOVA (PERMANOVA) were performed to compare differences in diazotrophic community composition among four meadows using Bray-Curtis dissimilarities in Hellinger-transformed relative abundances. Redundancy analysis (RDA) was conducted to test the relationships between diazotrophic community composition and environmental factors. The relationships between BNF rate, gene abundance, composition, and a-diversity of diazotrophs and environmental factors were explored using Pearson's correlation. Ordinary least-squares regression was conducted to test the relationship between the abundance of diazotrophic genera and the BNF rate. Structural equation modeling (SEM) and aggregated boosted tree (ABT) analyses were employed to explore the potential drivers of soil BNF rate. Before establishing the SEM, we eliminated the redundancy of soil and plant properties by removing the variables with high correlations (Spearman's $\rho^{2}>0.7$ ) using the VARCLUS program due to the strong collinearity among soil and plant properties (Martiny et al. 2011). Subsequently, $\mathrm{NH}_{4}{ }^{+}-\mathrm{N}^{-} \mathrm{NO}_{3}{ }^{-}-\mathrm{N}, \mathrm{AK}, \mathrm{pH}$, and $\mathrm{C} / \mathrm{N}$ were utilized to establish the SEM (Fig. S1). ABT analysis was conducted to quantify the relative importance of PFGs for BNF rates and diazotrophic community composition. All statistical analyses were performed with R v.3.6.2 (R core team) using the "ggplot2," "vegan," "Hmisc," "agricolae," "lavaan," and "gbmplus" packages.

\section{Results}

\subsection{Plant characteristics, soil properties, and BNF rates}

Meadow degradation strongly changed the composition of plant communities (Fig. S3 and Table 2). The plant cover decreased significantly along the degradation gradient and decreased most sharply in SD meadows with the lowest cover (39.4\%, Table 1). The BGB values of MD and SD were also significantly lower than those of ND and LD, although no significant difference was found between LD and ND. However, LD had the highest AGB value and lowest Chao1 index, whereas MD had the highest plant Shannon diversity and Chao1 indices. 
Table 1

Plant characteristics in four meadows along the degradation gradient. Values are means \pm standard error $(n=5)$. Different letters indicate significant differences between different treatments (Duncan's test, $P<0.05$ ). ND: non-degraded meadow; LD: lightly degraded meadow; MD: moderately degraded meadow; SD: severely degraded meadow. AGB: aboveground biomass; BGB: belowground biomass.

\begin{tabular}{|lllllll|}
\hline Parameters & ND & LD & MD & SD & $\boldsymbol{F}$ & $P$ \\
\hline Cover $(\%)$ & $94.20 \pm 1.24 \mathrm{a}$ & $83.00 \pm 1.55 \mathrm{~b}$ & $74.00 \pm 1.05 \mathrm{c}$ & $39.40 \pm 1.81 \mathrm{~d}$ & 268.69 & $<0.001$ \\
\hline AGB $\left(\mathrm{g} \cdot \mathrm{m}^{-2}\right)$ & $178.38 \pm 6.38 \mathrm{~b}$ & $253.28 \pm 15.03 \mathrm{a}$ & $167.35 \pm 10.64 \mathrm{~b}$ & $45.77 \pm 12.64 \mathrm{c}$ & 54.69 & $<0.001$ \\
\hline BGB $\left(\mathrm{g} \cdot \mathrm{m}^{-2}\right)$ & $\begin{array}{l}894.05 \pm 22.16 \\
\mathrm{a}\end{array}$ & $866.25 \pm 26.37 \mathrm{a}$ & $593.86 \pm 51.03 \mathrm{~b}$ & $158.98 \pm 40.06 \mathrm{c}$ & 86.17 & $<0.001$ \\
\hline $\begin{array}{l}\text { Shannon } \\
\text { diversity }\end{array}$ & $0.94 \pm 0.04 \mathrm{c}$ & $1.42 \pm 0.09 \mathrm{~b}$ & $1.77 \pm 0.04 \mathrm{a}$ & $1.64 \pm 0.08 \mathrm{a}$ & 31.64 & $<0.001$ \\
\hline \begin{tabular}{l} 
Richness \\
\hline
\end{tabular} & $8.20 \pm 0.49 \mathrm{~b}$ & $7.40 \pm 0.75 \mathrm{~b}$ & $12.20 \pm 0.37 \mathrm{a}$ & $8.20 \pm 0.37 \mathrm{~b}$ & 17.38 & $<0.001$ \\
\hline
\end{tabular}


Table 2

The characteristics of plant functional groups in four meadows along the degradation gradient. Values are means \pm standard error $(\mathrm{n}=5)$. Different letters indicate significant differences between different treatments (Duncan's test, $P<$

0.05). ND: non-degraded meadow; LD: lightly degraded meadow; MD: moderately degraded meadow; SD: severely degraded meadow; AGB: aboveground biomass; BGB: belowground biomass.

\begin{tabular}{|c|c|c|c|c|c|c|c|}
\hline Index & PFGs & ND & LD & MD & SD & $F$ & $P$ \\
\hline \multirow[t]{4}{*}{ Cover (\%) } & Sedge & $\begin{array}{l}72.33 \pm 4.15 \\
a\end{array}$ & $31.38 \pm 2.70 b$ & $23.18 \pm 2.22 c$ & $\begin{array}{l}4.25 \pm 1.18 \\
d\end{array}$ & 533.27 & $\begin{array}{l}<.001 \\
\end{array}$ \\
\hline & Grass & $\begin{array}{l}12.08 \pm 1.37 \\
b\end{array}$ & $35.36 \pm 3.72 a$ & $2.91 \pm 0.82 c$ & $\begin{array}{l}0.69 \pm 0.21 \\
c\end{array}$ & 306.84 & $<.001$ \\
\hline & Forbs & $8.18 \pm 1.51 \mathrm{~d}$ & $16.25 \pm 2.68 c$ & $46.52 \pm 2.62 \mathrm{a}$ & $\begin{array}{l}25.59 \pm \\
3.65 \mathrm{~b}\end{array}$ & 184.26 & $\begin{array}{l}<.001 \\
0.001\end{array}$ \\
\hline & Legume & $1.62 \pm 0.25 b$ & - & $1.40 \pm 0.50 \mathrm{~b}$ & $\begin{array}{l}8.87 \pm 0.78 \\
a\end{array}$ & 346.80 & $\begin{array}{l}<.001 \\
0.001\end{array}$ \\
\hline \multirow[t]{4}{*}{ AGB $\left(g \cdot m^{-2}\right)$} & Sedge & $\begin{array}{l}119.43 \pm \\
14.01 \mathrm{a}\end{array}$ & $\begin{array}{l}126.05 \pm \\
17.46 \mathrm{a}\end{array}$ & $64.76 \pm 5.79 b$ & $\begin{array}{l}1.72 \pm \\
0.71 \mathrm{c}\end{array}$ & 125.48 & $\begin{array}{l}<.001 \\
0.001\end{array}$ \\
\hline & Grass & $\begin{array}{l}43.34 \pm 9.05 \\
b\end{array}$ & $\begin{array}{l}86.14 \pm 12.34 \\
a\end{array}$ & $22.92 \pm 5.86 \mathrm{c}$ & $\begin{array}{l}4.48 \pm 1.25 \\
d\end{array}$ & 91.11 & $\begin{array}{l}<.001 \\
0.001\end{array}$ \\
\hline & Forbs & $\begin{array}{l}12.00 \pm 2.70 \\
d\end{array}$ & $41.09 \pm 6.04 b$ & $\begin{array}{l}75.59 \pm 13.75 \\
a\end{array}$ & $\begin{array}{l}30.32 \pm \\
4.22 \mathrm{c}\end{array}$ & 57.04 & $\begin{array}{l}<.001 \\
0.001\end{array}$ \\
\hline & Legume & $3.60 \pm 0.39 b$ & - & $4.09 \pm 2.71 b$ & $\begin{array}{l}9.25 \pm 2.20 \\
a\end{array}$ & 23.46 & $\begin{array}{l}<.001 \\
0.001\end{array}$ \\
\hline \multirow[t]{4}{*}{ BGB $\left(\mathrm{g} \cdot \mathrm{m}^{-2}\right)$} & Sedge & $\begin{array}{l}723.79 \pm \\
61.73 \mathrm{a}\end{array}$ & $\begin{array}{l}422.38 \pm \\
49.16 b\end{array}$ & $\begin{array}{l}277.52 \pm \\
48.94 \mathrm{c}\end{array}$ & $\begin{array}{l}15.40 \pm \\
5.45 \mathrm{~d}\end{array}$ & 201.99 & $\begin{array}{l}<.001 \\
0.001\end{array}$ \\
\hline & Grass & $\begin{array}{l}130.09 \pm \\
27.73 b\end{array}$ & $\begin{array}{l}294.55 \pm \\
39.26 a\end{array}$ & $\begin{array}{l}65.67 \pm 18.89 \\
c\end{array}$ & $\begin{array}{l}21.46 \pm \\
4.64 \mathrm{~d}\end{array}$ & 106.58 & $\langle .001$ \\
\hline & Forbs & $\begin{array}{l}30.69 \pm 6.32 \\
d\end{array}$ & $\begin{array}{l}149.32 \pm \\
22.67 \mathrm{~b}\end{array}$ & $\begin{array}{l}244.79 \pm \\
46.06 \mathrm{a}\end{array}$ & $\begin{array}{l}93.16 \pm \\
9.48 \mathrm{c}\end{array}$ & 59.70 & $\begin{array}{l}<.001 \\
0.001\end{array}$ \\
\hline & Legume & $9.48 \pm 2.20 \mathrm{~b}$ & - & $5.89 \pm 1.34 c$ & $\begin{array}{l}28.95 \pm \\
2.91 \mathrm{a}\end{array}$ & 208.32 & $\dot{0} .001$ \\
\hline \multirow[t]{4}{*}{ Shannon diversity } & Sedge & $0.31 \pm 0.08 b$ & $0.47 \pm 0.16 a$ & $0.36 \pm 0.01 a b$ & $\begin{array}{l}0.23 \pm 0.10 \\
b\end{array}$ & 4.50 & 0.018 \\
\hline & Grass & $0.22 \pm 0.03 b$ & $0.38 \pm 0.10 \mathrm{a}$ & $0.19 \pm 0.10 b$ & $\begin{array}{l}0.13 \pm 0.01 \\
b\end{array}$ & 10.85 & $\begin{array}{l}<.001 \\
0.001\end{array}$ \\
\hline & Forbs & $0.36 \pm 0.03 c$ & $0.57 \pm 0.10 b$ & $1.22 \pm 0.11 \mathrm{a}$ & $\begin{array}{l}1.16 \pm 0.10 \\
a\end{array}$ & 104.93 & $\begin{array}{l}<.001 \\
0.001\end{array}$ \\
\hline & Legume & $0.05 \pm 0.02 c$ & - & $0.01 \pm 0.00 \mathrm{~b}$ & $\begin{array}{l}0.13 \pm 0.04 \\
a\end{array}$ & 38.97 & $\begin{array}{l}<.001 \\
0.001\end{array}$ \\
\hline \multirow[t]{4}{*}{$\begin{array}{l}\text { Relative abundance } \\
\text { (\%) }\end{array}$} & Sedge & $\begin{array}{l}82.06 \pm 2.15 \\
a\end{array}$ & $48.36 \pm 6.09 b$ & $43.69 \pm 5.16 b$ & $\begin{array}{l}9.69 \pm 2.58 \\
c\end{array}$ & 233.95 & $\begin{array}{l}<.001 \\
0.001\end{array}$ \\
\hline & Grass & $6.62 \pm 0.77 b$ & $22.74 \pm 5.28 \mathrm{a}$ & $5.87 \pm 1.62 b$ & $\begin{array}{l}5.14 \pm 2.15 \\
b\end{array}$ & 39.95 & $\begin{array}{l}<.001 \\
\end{array}$ \\
\hline & Forbs & $\begin{array}{l}10.10 \pm 1.68 \\
d\end{array}$ & $28.90 \pm 4.33 c$ & $49.95 \pm 5.60 b$ & $\begin{array}{l}78.02 \pm \\
6.52 \mathrm{a}\end{array}$ & 178.13 & $\begin{array}{l}<.001 \\
0.001\end{array}$ \\
\hline & Legume & $1.22 \pm 0.07 b$ & - & $0.50 \pm 0.23 c$ & $\begin{array}{l}7.16 \pm 1.28 \\
a\end{array}$ & 129.93 & $\begin{array}{l}< \\
0.001\end{array}$ \\
\hline
\end{tabular}


Along the meadow degradation gradient, the dominant PFGs were transformed from sedges to forbs (Table 2). Sedge groups were dominant in ND and LD, with relative abundances of $82.06 \%$ and $48.36 \%$, respectively. Compared with ND, the plant cover, $A G B, B G B$, and relative abundance of sedge decreased significantly in degraded meadows. However, the relative abundance of forbs significantly increased during degradation and was highest in SD. The plant cover, AGB, BGB, and Shannon diversity of forbs were in the order of MD $>$ LD $>S D>N D$. The grass and forbs groups accounted for a minor proportion of the plants with a relative abundance of $5.14-22.74 \%$ and $0-7.16 \%$, respectively.

Degradation also caused deterioration of soil nutrient levels and BNF potential (Table 3). The contents of $\mathrm{NO}_{3}{ }^{-}-\mathrm{N}^{-}$and $\mathrm{NH}_{4}{ }^{+}-\mathrm{N}, \mathrm{DOC}, \mathrm{DON}, \mathrm{SOC}, \mathrm{TN}$, and C/N decreased significantly along the degradation gradient. Compared with ND, the contents of AP, TP, and SM dropped rapidly in MD and SD, but there was no significant difference between ND and LD. There was no significant difference in the AK content of four meadows. Soil pH showed a significant upward trend, but changed narrowly, ranging from 6.33 to 7.10 . Soil BNF rates decreased significantly along the degradation gradient, with a range of 17.34-79.84 $\mathrm{nmol} \mathrm{C}_{2} \mathrm{H}_{4} \mathrm{~g}^{-1}$ dry soil $\mathrm{d}^{-1}$ across all sites (Fig. 1A). The highest BNF was observed in ND and was 1.5-, 1.8-, and 4.6-fold higher than in LD, MD, and SD, respectively.

Table 3

Soil physicochemical properties in four meadows along the degradation gradient. Values are means \pm standard error ( $\mathrm{n}$ $=5$ ). Different letters indicate significant differences between different treatments (Duncan's test, $P<0.05$ ). ND: nondegraded meadow; LD: lightly degraded meadow; MD: moderately degraded meadow; SD: severely degraded meadow; AP: available P; DOC: dissolved organic C; DON: dissolved organic N; SOC: soil organic C; TN: total N; TP: total P; AK: available K; SM: soil moisture; $\mathrm{C} / \mathrm{N}$ : the ratio of SOC and TN.

\begin{tabular}{|lllllll|}
\hline Parameters & ND & LD & MD & SD & $F$ & $P$ \\
\hline $\mathrm{AP}\left(\mathrm{mg} \cdot \mathrm{kg}^{1}\right)$ & $2.29 \pm 0.17 \mathrm{a}$ & $2.18 \pm 0.17 \mathrm{a}$ & $1.67 \pm 0.13 \mathrm{~b}$ & $1.32 \pm 0.08 \mathrm{~b}$ & 10.17 & $<0.001$ \\
\hline $\mathrm{NO}_{3}{ }^{-} \mathrm{N}\left(\mathrm{mg} \cdot \mathrm{kg}^{-1}\right)$ & $13.05 \pm 1.07 \mathrm{a}$ & $6.74 \pm 0.21 \mathrm{~b}$ & $5.32 \pm 0.64 \mathrm{bc}$ & $3.69 \pm 0.47 \mathrm{c}$ & 36.98 & $<0.001$ \\
\hline $\mathrm{NH}_{4}{ }^{+} \mathrm{N}\left(\mathrm{mg} \cdot \mathrm{kg}^{-1}\right)$ & $6.97 \pm 0.73 \mathrm{a}$ & $3.49 \pm 0.35 \mathrm{~b}$ & $3.72 \pm 0.36 \mathrm{~b}$ & $3.29 \pm 0.45 \mathrm{~b}$ & 12.40 & $<0.001$ \\
\hline $\mathrm{DOC}\left(\mathrm{mg} \cdot \mathrm{kg}^{-1}\right)$ & $664.22 \pm 13.47 \mathrm{a}$ & $496.34 \pm 9.92 \mathrm{~b}$ & $427.84 \pm 9.56 \mathrm{c}$ & $327.48 \pm 7.73 \mathrm{~d}$ & 186.25 & $<0.001$ \\
\hline $\mathrm{DON}\left(\mathrm{mg} \cdot \mathrm{kg}^{-1}\right)$ & $195.66 \pm 7.65 \mathrm{a}$ & $147.46 \pm 8.19 \mathrm{~b}$ & $96.10 \pm 4.50 \mathrm{c}$ & $62.46 \pm 4.14 \mathrm{~d}$ & 83.83 & $<0.001$ \\
\hline $\mathrm{SOC}\left(\mathrm{g} \cdot \mathrm{kg}^{-1}\right)$ & $33.51 \pm 3.49 \mathrm{a}$ & $19.46 \pm 0.61 \mathrm{~b}$ & $13.57 \pm 0.41 \mathrm{c}$ & $10.94 \pm 0.88 \mathrm{c}$ & 30.02 & $<0.001$ \\
\hline $\mathrm{TN}\left(\mathrm{g} \cdot \mathrm{kg}^{-1}\right)$ & $2.42 \pm 0.19 \mathrm{a}$ & $1.77 \pm 0.05 \mathrm{~b}$ & $1.30 \pm 0.02 \mathrm{c}$ & $1.06 \pm 0.09 \mathrm{c}$ & 29.08 & $<0.001$ \\
\hline $\mathrm{TP}\left(\mathrm{g} \cdot \mathrm{kg}^{-1}\right)$ & $0.47 \pm 0.01 \mathrm{a}$ & $0.49 \pm 0.02 \mathrm{a}$ & $0.34 \pm 0.01 \mathrm{~b}$ & $0.30 \pm 0.03 \mathrm{~b}$ & 23.67 & $<0.001$ \\
\hline $\mathrm{AK}\left(\mathrm{mg} \cdot \mathrm{kg}^{-1}\right)$ & $116.96 \pm 11.19 \mathrm{a}$ & $106.23 \pm 4.48 \mathrm{a}$ & $95.52 \pm 10.38 \mathrm{a}$ & $95.98 \pm 6.54 \mathrm{a}$ & 1.39 & 0.280 \\
\hline $\mathrm{pH}$ & $6.43 \pm 0.04 \mathrm{c}$ & $6.65 \pm 0.03 \mathrm{ab}$ & $6.78 \pm 0.02 \mathrm{~b}$ & $6.90 \pm 0.07 \mathrm{a}$ & 21.14 & $<0.001$ \\
\hline $\mathrm{SM}(\%)$ & $23.35 \pm 1.19 \mathrm{a}$ & $22.88 \pm 0.55 \mathrm{a}$ & $18.37 \pm 0.57 \mathrm{~b}$ & $15.50 \pm 0.33 \mathrm{c}$ & 26.25 & $<0.001$ \\
\hline $\mathrm{C} / \mathrm{N}$ & $13.73 \pm 0.45 \mathrm{a}$ & $11.01 \pm 0.11 \mathrm{~b}$ & $10.46 \pm 0.23 \mathrm{~b}$ & $10.37 \pm 0.13 \mathrm{~b}$ & 34.74 & $<0.001$ \\
\hline
\end{tabular}

\subsection{Gene abundance and a-diversity of diazotrophs}

Meadow degradation significantly reduced the abundance of the nifH gene and the a-diversity of diazotrophs (Fig. 1B, C, and $\mathrm{D}$ ). The abundance of the nifH gene varied in the range of $0.99-7.68 \times 10^{6}$ copies $\cdot \mathrm{g}^{-1} \mathrm{dry}$ soil, with an average rate of $3.31 \times 10^{6}$ copies $\cdot g^{-1}$ dry soil. Compared with abundance of the nifH gene in ND, that in LD, MD, and SD decreased by $41.2 \%, 63.7 \%$, and $72.4 \%$, respectively. The Shannon and Chao 1 indices decreased by $22.1-25.1 \%$ and $16.0-33.3 \%$, 
respectively, in degraded meadows compared with those in ND, but no significant differences was observed among LD, $M D$, and SD.

The abundance of the nifH gene and the Shannon and Chao1 indices of diazotrophs were all positively correlated with AP, $\mathrm{NO}_{3}{ }^{-}-\mathrm{N}, \mathrm{NH}_{4}{ }^{+}-\mathrm{N}, \mathrm{DOC}, \mathrm{DON}, \mathrm{SOC}, \mathrm{TN}, \mathrm{SM}, \mathrm{AGB}, \mathrm{BGB}$, and plant cover, but negatively correlated with soil pH and plant Shannon diversity (Table 4). However, the AK and plant richness indices showed no significant correlation. TP showed positive correlation with the abundance of the nifH gene and the Shannon index of diazotrophs, but showed no significant correlation with the Chao1 diversity of diazotrophs. 
Table 4

Pearson's correlation between variables related to soil biological $\mathrm{N}_{2}$ fixation and environmental factors. Relationships between diazotrophic community composition and environmental factors were examined using Mantel test. Significant correlations with $P<0.05$ were shown in bold. AP: available P; DOC: dissolved organic C; DON: dissolved organic N; SOC: soil organic C; TN: total N; TP: total P; AK: available K; SM: soil moisture; C/N: the ratio of SOC and TN; AGB: aboveground biomass; BGB: belowground biomass; $H$ : plant Shannon diversity index.

\begin{tabular}{|c|c|c|c|c|c|c|c|c|c|c|}
\hline & \multicolumn{2}{|c|}{ BNF rate } & \multicolumn{2}{|c|}{$\begin{array}{l}\text { gene } \\
\text { abundance of } \\
\text { nifH }\end{array}$} & \multicolumn{2}{|c|}{$\begin{array}{l}\text { Shannon index } \\
\text { of diazotrophs }\end{array}$} & \multicolumn{2}{|c|}{$\begin{array}{l}\text { Chao1 index of } \\
\text { diazotrophs }\end{array}$} & \multicolumn{2}{|c|}{$\begin{array}{l}\text { Diazotrophic } \\
\text { community } \\
\text { composition }\end{array}$} \\
\hline & $r$ & $P$ & $r$ & $P$ & $r$ & $P$ & $r$ & $P$ & $r$ & $P$ \\
\hline AP & 0.748 & < 0.001 & 0.583 & $\hat{0.01}$ & 0.536 & $\hat{0.05}$ & 0.476 & $\begin{array}{l}<.05 \\
0.05\end{array}$ & 0.253 & <. 05 \\
\hline $\mathrm{NO}_{3}{ }^{-}-\mathrm{N}$ & 0.840 & <. 001 & 0.928 & $\hat{0.001}$ & 0.809 & <. 001 & 0.640 & $\begin{array}{l}<.01 \\
0.01\end{array}$ & 0.848 & $\hat{0.001}$ \\
\hline $\mathrm{NH}_{4}^{+}-\mathrm{N}$ & 0.663 & $\begin{array}{l}<.01 \\
0.01\end{array}$ & 0.578 & $\hat{0.01}$ & 0.722 & <. 001 & 0.481 & $\begin{array}{l}<.05 \\
0.05\end{array}$ & 0.606 & $\hat{0.001}$ \\
\hline DOC & 0.943 & $\hat{0.001}$ & 0.844 & $\hat{0.001}$ & 0.793 & $\hat{0.001}$ & 0.672 & $\hat{0.01}$ & 0.813 & $\hat{0.001}$ \\
\hline DON & 0.924 & <. 001 & 0.800 & $\hat{0} .001$ & 0.694 & $\hat{0.01}$ & 0.570 & $\dot{0.01}$ & 0.648 & $\hat{0} .001$ \\
\hline SOC & 0.836 & <. 001 & 0.839 & $\dot{0.001}$ & 0.780 & $\hat{0.001}$ & 0.550 & $\begin{array}{l}<.05 \\
0.05\end{array}$ & 0.795 & $\hat{0.001}$ \\
\hline $\mathrm{TN}$ & 0.861 & <. 001 & 0.810 & $\hat{0} .001$ & 0.753 & $\hat{0.001}$ & 0.516 & $\begin{array}{l}<.05 \\
0.05\end{array}$ & 0.720 & $\hat{0} .001$ \\
\hline TP & 0.766 & <. 001 & 0.626 & $\dot{0} 01$ & 0.578 & $\hat{0} 01$ & 0.393 & 0.086 & 0.245 & $\hat{0.05}$ \\
\hline AK & 0.395 & 0.085 & 0.304 & 0.129 & 0.369 & 0.110 & 0.225 & 0.340 & 0.212 & 0.057 \\
\hline $\mathrm{pH}$ & -0.845 & $\hat{0} .001$ & -0.839 & $\hat{0.001}$ & -0.595 & $\hat{0.01}$ & -0.571 & $\begin{array}{l}<.01 \\
0.01\end{array}$ & 0.588 & $\begin{array}{l}<.001 \\
0.00\end{array}$ \\
\hline SM & 0.827 & $\hat{0} .001$ & 0.607 & $\hat{0.01}$ & 0.556 & $\hat{0.05}$ & 0.395 & 0.085 & 0.321 & $\hat{0.01}$ \\
\hline $\mathrm{C} / \mathrm{N}$ & 0.777 & <. 001 & 0.852 & $\dot{0.001}$ & 0.784 & $\hat{0.001}$ & 0.610 & $\begin{array}{l}<.01 \\
0.01\end{array}$ & 0.851 & $\hat{0.001}$ \\
\hline Richness & -0.109 & 0.648 & -0.285 & 0.224 & -0.221 & 0.350 & 0.003 & 0.992 & -0.120 & 0.924 \\
\hline$H$ & -0.760 & <. 001 & -0.779 & $\hat{0.001}$ & -0.750 & $\hat{0.001}$ & -0.497 & $\begin{array}{l}<.05 \\
0.05\end{array}$ & 0.765 & $\hat{0.001}$ \\
\hline AGB & 0.669 & $\hat{0.01}$ & 0.390 & 0.089 & 0.303 & 0.194 & 0.458 & $\hat{0.05}$ & -0.040 & 0.622 \\
\hline BGB & 0.890 & 0.001 & 0.669 & $\hat{0} .01$ & 0.569 & $\dot{0} 01$ & 0.598 & $\dot{0.01}$ & 0.158 & 0.067 \\
\hline Cover & 0.950 & < 0.001 & 0.739 & $\begin{array}{l}< \\
0.001\end{array}$ & 0.589 & $\begin{array}{l}<.01 \\
0.01\end{array}$ & 0.594 & $\begin{array}{l}<.01 \\
0.01\end{array}$ & 0.309 & $\begin{array}{l}<.05 \\
0.05\end{array}$ \\
\hline
\end{tabular}

\subsection{Diazotrophic community composition}

At the phylum level, Proteobacteria and Cyanobacteria were the most abundant groups, accounting for $55.6 \%$ and $40.3 \%$, respectively, of the diazotrophs across all sites (Fig. 2A). Along the meadow degradation gradient, the relative abundance 
of Cyanobacteria increased, whereas that of Proteobacteria decreased. At the genus level, f_Nostocaceae (25.9\%), Scytonema (8.32\%), Nostoc (5.3\%), affiliated Cyanobacteria, Skermanella (20.2\%), o_Rhizobiales (12.4\%), C_Alphaproteobacteria (10.2\%), Scyronema (8.3\%), and affiliated Proteobacteria were the most abundant genera (Fig. 2B).

NMDS and Adonis analyses showed that diazotrophic community composition differed significantly along the degradation gradient (Fig. 3A). The diazotrophic community structure in ND showed obviously difference from that in degraded meadows, whereas the community structures of diazotrophs in LD, MD, and SD were similar. Nostocaceae (31.78\%)-affiliated with the order Nostocales-was the most abundant family, and its relative abundance increased along the gradient of meadow degradation (Fig. S3). The order of Rhizobiales showed a contrasting pattern to that of Nostocaceae (Fig. S1A), and its relative abundance was highest in ND and lowest in SD. The relative abundance of Skermanella-affiliated with the family Rhodospirillaceae and the order Rhizobiales-was highest in LD and lowest in ND (Fig. 2B and Fig. S1).

\subsection{Potential drivers of soil BNF rate along the meadow degradation gradient}

Diazotrophic community composition was significantly associated with all measured soil physicochemical properties (except for AK), plant cover, and Shannon diversity, and was most closely associated with $\mathrm{NO}_{3}{ }^{-} \mathrm{N}^{-}$and $\mathrm{C} / \mathrm{N}$ (Table 1). The relative abundances of Nostoc, Rhodopseudomonas, p_Proteobacteria, and o_Rhizobiales were positively correlated with AP, $\mathrm{NO}_{3}{ }^{-}-\mathrm{N}, \mathrm{NH}_{4}{ }^{+}-\mathrm{N}, \mathrm{DOC}, \mathrm{DON}, \mathrm{SOC}, \mathrm{TN}, \mathrm{TP}, \mathrm{SM}, \mathrm{C} / \mathrm{N}, \mathrm{BGB}$, and plant cover, but negatively correlated with soil pH and plant Shannon diversity (Fig. 4). However, the relative abundances of c_Alphaproteobacteria, f_Rhizobiales, and Skermanella showed an opposite pattern in response to soil and plant characteristics. Furthermore, Trichormus, Rhodomicrobium, and Scytonema showed no significant correlation with any of the soil and plant properties. RDA also confirmed that $A P, \mathrm{NO}_{3}{ }^{-}-\mathrm{N}$, $\mathrm{NH}_{4}{ }^{+}-\mathrm{N}, \mathrm{DOC}, \mathrm{DON}, \mathrm{SOC}, \mathrm{TN}, \mathrm{TP}, \mathrm{SM}, \mathrm{C} / \mathrm{N}, \mathrm{BGB}$, and plant cover were the main factors affecting diazotrophic community structure, rather than the $\mathrm{AK}, \mathrm{pH}$, plant richness, and Shannon diversity (Fig. 3B).

Soil BNF rate positively correlated with $\mathrm{AP} \mathrm{NO}_{3}{ }^{-}-\mathrm{N}, \mathrm{NH}_{4}{ }^{+}-\mathrm{N}, \mathrm{DOC}, \mathrm{DON}, \mathrm{SOC}, \mathrm{TN}, \mathrm{TP}, \mathrm{SM}, \mathrm{C} / \mathrm{N}, \mathrm{AGB}, \mathrm{BGB}$, and plant cover, but negatively correlated with soil $\mathrm{pH}$ and plant Shannon diversity (Table 4). However, AK and the plant richness index showed no significant correlation with soil BNF rate. Soil BNF rate was also significantly associated with diazotrophic community composition (Mantel test: $r=0.66, P<0.001$ ), and increased with the absolute abundance of dominant diazotrophic groups, including o_Rhizobiales, Scytonema, p_Proteobacteria, Nostoc, and Rhodopseudomonas (Fig. 5 and Table 5). 
Table 5

Pearson's correlation test between dominant diazotrophic genus and the rate of $\mathrm{N}_{2}$ fixation $(\mathrm{n}=20)$. Significant correlations with $P<0.05$ were shown in bold.

\begin{tabular}{|lllll|}
\hline Diazotroph taxa & \multicolumn{3}{l}{ Absolute abundance } & \multicolumn{2}{l|}{ Relative abundance } \\
\cline { 2 - 6 } & $\mathbf{r}$ & $\boldsymbol{P}$ & $\mathbf{r}$ & $\boldsymbol{P}$ \\
\hline f_Nostocaceae & 0.349 & 0.120 & -0.823 & $<0.001$ \\
\hline Skermanella & -0.116 & 0.332 & -0.479 & 0.210 \\
\hline O_Rhizobiales & $\mathbf{0 . 8 0 4}$ & $<0.001$ & $\mathbf{0 . 8 0 4}$ & $<0.01$ \\
\hline C_Alphaproteobacteria & 0.266 & 0.241 & -0.724 & $<0.001$ \\
\hline Scytonema & $\mathbf{0 . 7 3 5}$ & $<0.01$ & -0.061 & 0.818 \\
\hline p_Proteobacteria & $\mathbf{0 . 7 6 1}$ & $<0.001$ & $\mathbf{0 . 7 3 3}$ & $<0.01$ \\
\hline Nostoc & $\mathbf{0 . 6 3 7}$ & $<0.01$ & $\mathbf{0 . 4 5 4}$ & $\mathbf{0 . 0 4 4}$ \\
\hline Rhodopseudomonas & $\mathbf{0 . 6 6 8}$ & $<0.001$ & $\mathbf{0 . 7 1 9}$ & $<0.001$ \\
\hline Rhodomicrobium & 0.015 & 0.915 & -0.422 & 0.058 \\
\hline Trichormus & 0.492 & $<0.05$ & 0.719 & 0.825 \\
\hline
\end{tabular}

The established SEM explained $98 \%$ of the variance in the BNF rate (Fig. 6), and the plant community had a direct effect on BNF by altering the plant cover. In addition, the plant community affected the soil $\mathrm{NH}_{4}{ }^{+}-\mathrm{N}_{1} \mathrm{NO}_{3}{ }^{-} \mathrm{N}$, and $\mathrm{C} / \mathrm{N}$, diazotrophic composition, and Shannon diversity, and further regulated the soil BNF rate (Fig. 6A). The plant cover had the highest total effect on soil BNF rates (Fig. 6B). Given the predominant regulation of soil BNF rate by plants, the effects of specific PFGs on soil BNF rate and the structure of the diazotrophic community were further examined by ABT modeling (Fig. 7). The plant cover of sedges was the main driver for soil BNF rate, gene abundance, a-diversity, and diazotrophic community composition. In total, the sedge groups had a major effect on soil BNF rate, which together accounted for $58.5 \%$ of the variation (Fig. 7A). The sedge groups were also the main factors affecting the gene abundance, composition, and diversity of diazotrophs (Fig. 7B). In addition, the forbs groups also had an important influence on soil $\mathrm{N}_{2}$ fixation and related diazotrophic community structure, accounting for $21-39.83 \%$ of the variation. However, legumes and grass groups had little effect on soil BNF and diazotrophic community structure.

\section{Discussion}

Our results showed that the BNF rate was significantly higher in ND (1.5-4.6-fold) than in degraded meadows, suggesting that alpine meadow degradation depressed soil BNF potential on the Tibetan Plateau (Fig. 1). Similar results were also found in the processes of vegetation restoration or degradation, in which the soil BNF potential increased or decreased, respectively, in various ecosystems (Li et al. 2021; Lopez-Lozano et al. 2016; Zhang et al. 2020b). For example, during the degradation of alpine peatlands, the soil BNF rates decreased by $42.7 \%$ from pristine marshes dominated by sedge (1.71 $\left.\mu \mathrm{mol} \mathrm{N} \cdot \mathrm{g}^{-1} \cdot \mathrm{d}^{-1}\right)$ to moderately degraded meadows $\left(0.98 \mu \mathrm{mol} \mathrm{N} \cdot \mathrm{g}^{-1} \cdot \mathrm{d}^{-1}\right)$, and decreased by $88.3 \%$ in sandy meadows $\left(0.20 \mu \mathrm{mol} \mathrm{N} \cdot \mathrm{g}^{-1} \cdot \mathrm{d}^{-1}\right)$ (Zhang et al. 2020b). In an abandoned farmland ecosystem, the BNF rates increased during restoration and ranged from 323.9 to $654.1 \mathrm{nmol} \mathrm{C}{ }_{2} \mathrm{H}_{4} \mathrm{~g}^{-1}$ dry soil d $\mathrm{d}^{-1}$ (Li et al. 2021), which was approximately 10-fold higher than that in our study. In addition, soil BNF rates in alpine meadow ecosystems were lower than those reported in most agro-ecosystems, but higher than those reported in degraded grasslands and forests with human impacts (Horel et al. 2018; Li et al. 2021; Wu et al. 2021). This difference may be attributed to the various plant-soil-microbe interactions in different environments. 
In our study, plant community structure was the primary factor driving the variation of soil BNF potential, and plant cover was observed to have the greatest effect on soil BNF rates in SEM (Fig. 6). Furthermore, the sedge groups had a major effect on the variation in soil BNF rates (Fig. 7). Plant cover and biomass (above- and belowground) were significantly reduced along the degradation gradient (Table 1\&2), suggesting that meadow degradation reduced aboveground productivity and destroyed the plant community structure. Similar results were found by Luo et al. (2018b), who found that grassland degradation dramatically reduced plant cover and productivity and confirmed that PFG characteristics are useful for indicating the process of land degradation. In this study, the dominant PFGs shifted from sedges in ND to forbs in SD (Table 2). One possible reason is that compared to other PFGs, forbs are more competitive with resources in severely degraded alpine meadows-especially in the Tibetan meadow with extensive N limitation (Zhang et al. 2020a). In addition, the variation in PFGs can substantially trigger changes in soil physicochemical properties.

The variables associated with plant characteristics can have profound impacts the soil-diazotroph interactions by direct or indirect effects, and consequently regulate the soil BNF potential (Lopez-Lozano et al. 2016; Zhang et al. 2020b). A lower plant biomass indicates a smaller rhizosphere volume and a lower supply of root exudates (i.e., labile organic $\mathrm{C}$ and $\mathrm{N}$ ) for diazotrophs, which are detrimental to the function of BNF (Yang et al. 2021; Zhang et al. 2020b). Indeed, we observed obvious effects of meadow degradation on the diazotrophic community composition (Fig. 3). The abundance of nifH gene and a-diversity were all significantly declined along the degradation gradient (Fig. 1). Our results are consistent with those of other studies showing that the absolute abundance and diversity of diazotrophs are reduced by grassland degradation and increase with restoration (Lopez-Lozano et al. 2016; Wang et al. 2017). A possible reason for this is that meadow degradation not only reduces soil nutrition level and nutrient availability, but also provides a worse living environment for diazotrophs and further inhibits their growth (Zhang et al. 2020a). PFGs may have a stronger influence on soil microbial diversity and soil function by providing more carbon resources (Andruschkewitsch et al. 2014; Wang et al. 2021a). In our study, the sedge groups had a major effect on the soil BNF rate, abundance of the nifH gene, the Shannon diversity index, and community composition of diazotrophs (Fig. 7). The roots and rhizomes of sedges are frequently found to promote diazotroph growth (Rejmankova et al. 2018). In addition, the weak influence of legumes on soil BNF rates and diazotrophic community structure suggests that free-living diazotrophs contributed more than symbiotic diazotrophs to BNF (Zhang et al. 2020b). Therefore, plant-diazotroph interactions may be the major controlling factor in determining BNF potential during alpine meadow degradation-especially for sedges rather than legumes.

The plant biomass indirectly determines the input of organic matter and other effective substrates into the soil through the decomposition of plant litter and root residue (Yang et al. 2021). In our study, the soil moisture and nutrient supply capacity in the surface layers decreased significantly along the meadow degradation gradient (Table 3). Recent studies of Tibetan alpine meadows have shown that meadow degradation is accompanied by a sharp decline in soil nutrients and moisture. For instance, the contents of SOC and TN in a pristine meadow were seven and six times higher, respectively, than those in a sandy meadow (Zhang et al. 2020b) The decrease in soil moisture during meadow degradation could be attributed to the decrease in vegetation cover or other traits (e.g., root biomass, leaf area, and plant height) that can increase evaporation and reduce soil water retention capacity (Yang et al. 2013). The decrease in aboveground and belowground plant biomass in degraded meadows could reduce the input of organic matter into the soil through decomposition of litter and release of root exudates (Prieto et al. 2017).

We found that all measured soil properties (except AK) were strongly correlated with BNF rates and diazotrophic community composition (Table 4). Both the abundance of the nifH gene and a-diversity of diazotrophs were positively correlated with soil nutrients (e.g., $\mathrm{AP}, \mathrm{NO}_{3}{ }^{-}{ }^{-} \mathrm{N}, \mathrm{NH}_{4}{ }^{+}-\mathrm{N}, \mathrm{DOC}, \mathrm{SOC}$, and TP) in our study. Particularly, DOC and DON had higher correlation coefficients with BNF rates. Dissolved organic matter, which can supply these microbes with additional available organic nutrients, is the major controlling factor for $\mathrm{N}$ cycling in soils (Levy-Booth et al. 2014). $\mathrm{N}_{2}$ fixation by heterotrophic diazotrophs is highly dependent on organic substances from the environment, such as soil and plant systems; thus, it is necessary to maintain a high $\mathrm{N}_{2}$-fixing ability in substrates with high C quantities (Dai et al. 2021). In

Page 14/26 
addition, the reaction of BNF is catalyzed by nitrogenase with high energy consumption, suggesting that the growth and functions of diazotrophs are not only controlled by C availability, but also by N, P, and K availability (Zheng et al. 2019). However, several studies have shown that high inorganic $\mathrm{N}$ contents (e.g., $\mathrm{NH}_{4}{ }^{+}-\mathrm{N}$ and $\mathrm{NO}_{3}{ }^{-}{ }^{-} \mathrm{N}$ ) significantly inhibit soil BNF capacity in agro-ecosystems with $\mathrm{N}$ fertilization addition (Dai et al. 2021; Fan et al. 2019), which is inconsistent with our results. A possible reason for this difference is that the soil $\mathrm{N}$ concentration tended to be higher in the other studies, resulting in excess $\mathrm{N}$ content limiting the growth and activity of microorganisms (Wang et al. 2018). In our study, the increase in soil $\mathrm{N}$ concentration may have been the result of improved BNF capacity, rather than its cause. This has also been observed by Zhang et al. (2020b) in a degraded alpine peatland on the Tibetan Plateau. In addition, soil moisture content and $\mathrm{pH}$ are also important factors for soil BNF rate, as they affect soil microbial activity and determine the processes of $\mathrm{N}_{2}$ fixation (Wang et al. 2018; Zhang et al. 2020b). Zhang et al. (2020b) found that the decreased soil BNF potential in a degraded alpine peatland was primarily driven by soil moisture, which led to changes in soil physicochemical properties and plant community, further leading to changes in soil BNF. In this study, although there was a positive correlation between soil moisture and BNF rate (Table 4), the range of variation (15.50-23.35\%) of soil moisture was too narrow to cause changes in the vegetation community; thus, it was not the primary driver of soil BNF in the process of alpine meadow degradation. Wang et al. (2017) reported that soil pH was a major driver of diversity and turnover of diazotrophic community in alpine meadows on the Tibetan Plateau. However, $\mathrm{pH}$ had a weak influence on the soil BNF rate and diazotrophic community in our study (Table 4). The reason for this difference is that the influence of $\mathrm{pH}$ on the microbial community is scale-dependent (Martiny et al. 2011). The significant effect of soil pH on BNF can be determined at a larger scale with an obvious $\mathrm{pH}$ gradient, such as the changes in $\mathrm{pH}$ in an altitudinal gradient.

In our study, both heterotrophic and autotrophic diazotrophs, including Proteobacteria and Cyanobacteria, performed the majority of $\mathrm{N}_{2}$ fixation in the four meadows (Fig. 2). More specifically, these sequences were identified as members of the orders Nostocales, Rhodospirillales, and Rhizobiale (Fig. S3). However, their responses to meadow degradation varied, indicating differences in substrate preference and nutrient acquisition strategies (Zhang et al. 2016). The relative abundance of Proteobacteria decreased, and that of Cyanobacteria increased along the meadow degradation gradient (Fig. 2). Meadow degradation affects diazotrophic composition by altering various soil and plant properties, such as $\mathrm{C} / \mathrm{N}$, SOC, and DOC, which are the most important factors in shaping the diazotrophic community composition (Table 4). Previous studies have also demonstrated that $\mathrm{C}$ substrates are the main drivers of soil microbial community composition (Yang et al. 2021; Zhang et al. 2016). Indeed, both f_Nostocaceae and Skermanella-two of the most dominant abundant genera-were significantly correlated with soil C/N, SOC, and DOC (Fig. 4). The reduced input of labile organic C from plants caused by meadow degradation may further limit the growth of dominant diazotrophic taxa, especially heterotrophic diazotrophs (Che et al. 2019). The increase in the abundance of autotrophic diazotrophs with meadow degradation can be explained by the higher light availability caused by the decreased plant cover (Zhang et al. 2020b). However, the relative contribution of heterotrophic and autotrophic diazotrophs to soil BNF is unclear, and further research is needed.

Although the $\mathrm{nifH}$ gene may not be expressed to produce nitrogenase and the enzyme activity is also affected by various factors (Mårtensson et al. 2009; Wang et al. 2018), nifH gene abundance can still be used as a sensitive bioindicator of soil BNF capacity, as shown in our study (Li et al. 2021). In addition, the abundance of diazotrophic genera, including Nostoc, Scytonema, Rhodopseudomonas, o_Rhizobiales, and p_Proteobacteria, is more indicative of soil BNF rates than their relative abundance (Table 5 and Fig. 5), which confirms the importance of the nifH gene. Furthermore, the Shannon diversity of diazotrophs was also suitable for predicting and characterizing the BNF rate (Fig. 6). Loss of population and diversity of soil microorganisms can destroy multiple ecosystem functions, such as the maintenance of soil fertility and health (Wagg et al. 2014). Therefore, communities harboring diverse diazotrophic taxa can provide more ecosystem functions than those with fewer species. Degraded alpine meadows may be particularly sensitive to the functional deterioration of BNF, as its main N source is BNF. Additionally, decreased BNF and an altered community composition of

Page 15/26 
diazotrophs may be coupled with other biogeochemical processes-such as greenhouse gas production-that pose a threat to climate change (Eberhard et al. 2018).

\section{Conclusions}

Our results suggest that alpine meadow degradation significantly decreased diazotrophic abundance, including Nostoc, Scytonema, Rhodopseudomonas, Rhizobiales, and Proteobacteria, and thus depressed the soil BNF rates. The reduced soil BNF was closely related to changes in the plant community (especially in the sedges family) that regulates soil moisture, nutrient levels (dissolved organic $\mathrm{C}$ and $\mathrm{N}$ ), nifH gene abundance, and diazotrophic community composition. Both autotrophic (Cyanobacteria) and heterotrophic (Proteobacteria) diazotrophs contributed to BNF. Our results emphasize the importance of plant functional groups in shaping the diazotrophic community and regulating soil BNF potential, and provide insights into the underlying mechanism of soil BNF in alpine meadows.

\section{Declarations}

\section{Funding}

This work was financially supported by the National Natural Sciences Foundation of China (41761008); the Natural Science Basic Research Program of Shaanxi Province (2019KJXX-081; 2021JM-605); Chinese Universities Scientific Fund (2452018336).

\section{Conflicts of interest/Competing interests}

There are no conflicts of interest or competing interest.

\section{Availability of data and material}

All raw sequences were submitted to the NCBI Sequence Read Archive database (No. SRP318670). Other data is available by request to the authors.

\section{Code availability}

Not applicable

\section{Authors' contributions}

Lu Zhang: Conceptualization, Methodology, Investigation, Writing - original draft. Xiangtan Wang: Investigation, Funding acquisition. Jie Wang: Investigation, Conceptualization, Formal analysis. Lirong Liao: Methodology, Visualization. Lei Shilong: Data analyzation. Guobin Liu: Investigation, Funding acquisition. Chao Zhang: Conceptualization, Supervision, Writing - review \& editing, Funding acquisition.

\section{References}

Andruschkewitsch M, Wachendorf C, Sradnick A, Hensgen F, Joergensen RG, Wachendorf M (2014) Soil substrate utilization pattern and relation of functional evenness of plant groups and soil microbial community in five low mountain NATURA 2000. Plant Soil 383: 275-289. https://doi.org/10.1007/s11104-014-2167-9

Che R, Deng Y, Wang F, Wang W, Xu Z, Hao Y, Xue K, Zhang B, Tang L, Zhou H, Cui X (2018) Autotrophic and symbiotic diazotrophs dominate nitrogen-fixing communities in Tibetan grassland soils. Sci Total Environ 639: 997-1006. https://doi.org/10.1016/j.scitotenv.2018.05.238 
Che R, Wang Y, Li K, Xu Z, Hu J, Wang F, Rui Y, Li L, Pang Z, Cui X (2019) Degraded patch formation significantly changed microbial community composition in alpine meadow soils. Soil Tillage Res 195.

https://doi.org/10.1016/j.still.2019.104426

Che RX, Wang F, Wang WJ, Zhang J, Zhao X, Rui YC, Xu ZH, Wang YF, Hao YB, Cui XY (2017) Increase in ammoniaoxidizing microbe abundance during degradation of alpine meadows may lead to greater soil nitrogen loss. Biogeochemistry 136: 341-352. https://doi.org/10.1007/s10533-017-0399-5

Colin Y, Goberna M, Verdú M, Navarro-Cano JA (2019) Successional trajectories of soil bacterial communities in mine tailings: The role of plant functional traits. J Environ Manage 241: 284-292.

https://doi.org/10.1016/j.jenvman.2019.04.023

Dai X, Song D, Guo Q, Zhou W, Liu G, Ma R, Liang G, He P, Sun G, Yuan F, Liu Z (2021) Predicting the influence of fertilization regimes on potential $\mathrm{N}$ fixation through their effect on free-living diazotrophic community structure in double rice cropping systems. Soil Biol Biochem 156: 108220. https://doi.org/10.1016/j.soilbio.2021.108220

Eberhard EK, Marcarelli AM, Baxter CV (2018) Co-occurrence of in-stream nitrogen fixation and denitrification across a nitrogen gradient in a western U.S. watershed. Biogeochemistry 139: 179-195. https://doi.org/10.1007/s10533-018-0461-y

Edgar RC (2010) Search and clustering orders of magnitude faster than BLAST. Bioinformatics 26: 2460-2461. https://doi.org/10.1093/bioinformatics/btq461

Fan K, Delgado-Baquerizo M, Guo X, Wang D, Wu Y, Zhu M, Yu W, Yao H, Zhu YG, Chu H (2019) Suppressed N fixation and diazotrophs after four decades of fertilization. Microbiome 7: 143. https://doi.org/10.1186/s40168-019-0757-8

Fish JA, Chai BL, Wang Q, Sun YN, Brown CT, Tiedje JM, Cole JR (2013) FunGene: the functional gene pipeline and repository. Front Microbiol 4: 14. https://doi.org/10.3389/fmicb.2013.00291

Fu G, Shen Z, Zhang X, Zhou Y, Zhang Y (2012) Response of microbial biomass to grazing in an alpine meadow along an elevation gradient on the Tibetan Plateau. Eur J Soil Biol 52: 27-29. https://doi.org/10.1016/j.ejsobi.2012.05.004

Horel A, Potyo I, Szili-Kovacs T, Molnar S (2018) Potential nitrogen fixation changes under different land uses as influenced by seasons and biochar amendments. Arab J Geosci 11. https://doi.org/10.1007/s12517-018-3916-5

Huang LN, Tang FZ, Song YS, Wan CY, Wang SL, Liu WQ, Shu WS (2011) Biodiversity, abundance, and activity of nitrogenfixing bacteria during primary succession on a copper mine tailings. FEMS Microbiol Ecol 78: 439-450. https://doi.org/10.1111/j.1574-6941.2011.01178.x

Kou D, Ding J, Li F, Wei N, Fang K, Yang G, Zhang B, Liu L, Qin S, Chen Y, Xia J, Yang Y (2019) Spatially-explicit estimate of soil nitrogen stock and its implication for land model across Tibetan alpine permafrost region. Sci Total Environ 650: 1795-1804. https://doi.org/10.1016/j.scitotenv.2018.09.252

Kumar U, Panneerselvam P, Govindasamy V, Vithalkumar L, Senthilkumar M, Banik A, Annapurna K (2017) Long-term aromatic rice cultivation effect on frequency and diversity of diazotrophs in its rhizosphere. Ecol Eng 101: 227-236. https://doi.org/10.1016/j.ecoleng.2017.02.010

Levy-Booth DJ, Prescott CE, Grayston SJ (2014) Microbial functional genes involved in nitrogen fixation, nitrification and denitrification in forest ecosystems. Soil Biol Biochem 75: 11-25. https://doi.org/10.1016/j.soilbio.2014.03.021

Li DD, Zhang XY, Dungait JAJ, Wen XF, Quine TA, Wang QB (2021) Changes in the biological N-2-fixation rates and diazotrophic community as vegetation recovers on abandoned farmland in a karst region of China. Appl Soil Ecol 158: 8.

Page $17 / 26$ 
https://doi.org/10.1016/j.apsoil.2020.103808

Lindstroem K, Mousavi SA (2020) Effectiveness of nitrogen fixation in rhizobia. Microb Biotechnol 13: 1314-1335. https://doi.org/10.1111/1751-7915.13517

Lopez-Lozano NE, Carcano-Montiel MG, Bashan Y (2016) Using native trees and cacti to improve soil potential nitrogen fixation during long-term restoration of arid lands. Plant Soil 403: 317-329. https://doi.org/10.1007/s11104-016-2807-3

Louca S, Polz MF, Mazel F, Albright MBN, Huber JA, O'Connor MI, Ackermann M, Hahn AS, Srivastava DS, Crowe SA, Doebeli M, Parfrey LW (2018) Function and functional redundancy in microbial systems. Nat Ecol Evol 2: 936-943. https://doi.org/10.1038/s41559-018-0519-1

Luo J, Liu X, Yang J, Liu Y, Zhou J (2018) Variation in plant functional groups indicates land degradation on the Tibetan Plateau. Sci Rep 8: 17606. https://doi.org/10.1038/s41598-018-36028-5

Mårtensson L, Díez B, Wartiainen I, Zheng W, El-Shehawy R, Rasmussen U (2009) Diazotrophic diversity, nifH gene expression and nitrogenase activity in a rice paddy field in Fujian, China. Plant Soil 325: 207.

https://doi.org/10.1007/s11104-009-9970-8

Martiny JBH, Eisen JA, Penn K, Allison SD, Horner-Devine MC (2011) Drivers of bacterial $\beta$-diversity depend on spatial scale. Proc Natl Acad Sci U S A 108: 7850. https://doi.org/10.1073/pnas.1016308108

Patra AK, Abbadie L, Clays-Josserand A, Degrange V, Grayston SJ, Guillaumaud N, Loiseau P, Louault F, Mahmood S, Nazaret S, Philippot L, Poly F, Prosser JI, Le Roux X (2006) Effects of management regime and plant species on the enzyme activity and genetic structure of $\mathrm{N}$-fixing, denitrifying and nitrifying bacterial communities in grassland soils. Environ Microbio 8: 1005-1016. https://doi.org/10.1111/j.1462-2920.2006.00992.x

Perez-Montano F, Alias-Villegas C, Bellogin RA, del Cerro P, Espuny MR, Jimenez-Guerrero I, Lopez-Baena FJ, Ollero FJ, Cubo $\mathrm{T}$ (2014) Plant growth promotion in cereal and leguminous agricultural important plants: From microorganism capacities to crop production. Microbiol Res 169: 325-336. https://doi.org/10.1016/j.micres.2013.09.011

Prieto I, Birouste M, Zamora-Ledezma E, Gentit A, Goldin J, Volaire F, Roumet C (2017) Decomposition rates of fine roots from three herbaceous perennial species: combined effect of root mixture composition and living plant community. Plant Soil 415: 359-372. https://doi.org/10.1007/s11104-016-3163-z

Reed SC, Cleveland CC, Townsend AR (2011) Functional Ecology of Free-Living Nitrogen Fixation: A Contemporary Perspective. In: DJ Futuyma, HB Shaffer, D Simberloff (eds) Annual Review of Ecology, Evolution, and Systematics, Vol 42. Annual Reviews, Palo Alto.

Rejmankova E, Sirova D, Castle ST, Barta J, Carpenter H (2018) Heterotrophic N-2-fixation contributes to nitrogen economy of a common wetland sedge, Schoenoplectus californicus. Plos One 13. https://doi.org/10.1371/journal.pone.0195570

Rosch C, Bothe H (2005) Improved assessment of denitrifying, N-2-fixing, and total-community bacteria by terminal restriction fragment length polymorphism analysis using multiple restriction enzymes. Appl and Environ Microbiol 71: 2026-2035. https://doi.org/10.1128/aem.71.4.2026-2035.2005

Smercina DN, Evans SE, Friesen ML, Tiemann LK (2019) To Fix or Not To Fix: Controls on Free-Living Nitrogen Fixation in the Rhizosphere. Appl and Environ Microbiol 85: 14. https://doi.org/10.1128/aem.02546-18

Urak I, Hartel T, Galle R, Balog A (2017) Worldwide peatland degradations and the related carbon dioxide emissions: the importance of policy regulations. Environ Sci Policy 69: 57-64. https://doi.org/10.1016/j.envsci.2016.12.012

Page $18 / 26$ 
Vicente EJ, Dean DR (2017) Keeping the nitrogen-fixation dream alive. Proc Natl Acad Sci U S A 114: 3009-3011. https://doi.org/10.1073/pnas.1701560114

Wagg C, Bender SF, Widmer F, van der Heijden MGA (2014) Soil biodiversity and soil community composition determine ecosystem multifunctionality. Proc Natl Acad Sci U S A 111: 5266-5270. https://doi.org/10.1073/pnas.1320054111

Wang C, Zheng MM, Shen RF (2020a) Diazotrophic communities are more responsive to maize cultivation than phosphorus fertilization in an acidic soil. Plant Soil 452: 499-512. https://doi.org/10.1007/s11104-020-04596-z

Wang J, Wang X, Liu G, Wang G, Zhang C (2021a) Grazing-to-fencing conversion affects soil microbial composition, functional profiles by altering plant functional groups in a Tibetan alpine meadow. Appl Soil Ecol 166. https://doi.org/10.1016/j.apsoil.2021.104008

Wang J, Wang X, Liu G, Zhang C, Wang G (2021b) Bacterial richness is negatively related to potential soil multifunctionality in a degraded alpine meadow. Ecol Indic 121: 106996. https://doi.org/10.1016/j.ecolind.2020.106996 Wang J, Wang XT, Liu GB, Wang GL, Wu Y, Zhang C (2020b) Fencing as an effective approach for restoration of alpine meadows: Evidence from nutrient limitation of soil microbes. Geoderma 363: 10.

https://doi.org/10.1016/j.geoderma.2019.114148

Wang Q, Wang J, Li Y, Chen D, Ao J, Zhou W, Shen D, Li Q, Huang Z, Jiang Y (2018) Influence of nitrogen and phosphorus additions on N2-fixation activity, abundance, and composition of diazotrophic communities in a Chinese fir plantation. Sci Total Environ 619-620: 1530-1537. https://doi.org/10.1016/j.scitotenv.2017.10.064

Wang Y, Li C, Kou Y, Wang J, Tu B, Li H, Li X, Wang C, Yao M (2017) Soil pH is a major driver of soil diazotrophic community assembly in Qinghai-Tibet alpine meadows. Soil Biol Biochem 115: 547-555. https://doi.org/10.1016/j.soilbio.2017.09.024

Wang Y, Li C, Tu B, Kou Y, Li X (2021c) Species pool and local ecological assembly processes shape the $\beta$-diversity of diazotrophs in grassland soils. Soil Biol Biochem 160: 108338. https://doi.org/10.1016/j.soilbio.2021.108338

Wen L, Dong SK, Zhu L, Li XY, Shi JJ, Wang YL, Ma YS (2010) The construction of Grassland Degradation Index for Alpine Meadow in Qinghai-Tibetan Plateau. In: Z Yang, B Chen (eds) International Conference on Ecological Informatics and Ecosystem Conservation.

Wu C, Wei X, Hu Z, Liu Y, Hu Y, Qin H, Chen X, Wu J, Ge T, Zhran M, Su Y (2021) Diazotrophic Community Variation Underlies Differences in Nitrogen Fixation Potential in Paddy Soils Across a Climatic Gradient in China. Microb Ecol 81: 425-436. https://doi.org/10.1007/s00248-020-01591-w

Wu G-L, Huang Z, Liu Y-F, Cui Z, Liu Y, Chang X, Tian F-P, López-Vicente M, Shi Z-H (2019) Soil water response of plant functional groups along an artificial legume grassland succession under semi-arid conditions. Agric For Meteorol 278. https://doi.org/10.1016/j.agrformet.2019.107670

Yang L, Rees RM, Nie J, Xu C, Cao W (2021) Carbon substrates exert a stronger role than mineral nitrogen application in structuring soil diazotroph communities during Chinese milk vetch growth. Appl Soil Ecol 158: 103778. https://doi.org/10.1016/j.apsoil.2020.103778

Yang Z-p, Gao J-x, Zhao L, Xu X-I, Ouyang H (2013) Linking thaw depth with soil moisture and plant community composition: effects of permafrost degradation on alpine ecosystems on the Qinghai-Tibet Plateau. Plant Soil 367: 687700. https://doi.org/10.1007/s11104-012-1511-1 
Zhang C, Liu G, Song Z, Wang J, Guo L (2018) Interactions of soil bacteria and fungi with plants during long-term grazing exclusion in semiarid grasslands. Soil Biol Biochem 124: 47-58. https://doi.org/10.1016/j.soilbio.2018.05.026

Zhang C, Liu G, Xue S, Wang G (2016) Soil bacterial community dynamics reflect changes in plant community and soil properties during the secondary succession of abandoned farmland in the Loess Plateau. Soil Biol Biochem 97: 40-49. https://doi.org/10.1016/j.soilbio.2016.02.013

Zhang R, Degen AA, Bai YF, Zhang T, Wang XM, Zhao XY, Shang ZH (2020a) The forb, Ajania tenuifolia, uses soil nitrogen efficiently, allowing it to be dominant over sedges and Graminae in extremely degraded grasslands: Implications for grassland restoration and development on the Tibetan Plateau. Land Degrad Dev 31: 1265-1276.

https://doi.org/10.1002/ldr.3555

Zhang X, Jia X, Wu H, Li J, Yan L, Wang J, Li Y, Kang X (2020b) Depression of soil nitrogen fixation by drying soil in a degraded alpine peatland. Sci Total Environ 747: 141084. https://doi.org/10.1016/j.scitotenv.2020.141084

Zheng MH, Zhou ZH, Luo YQ, Zhao P, Mo JM (2019) Global pattern and controls of biological nitrogen fixation under nutrient enrichment: A meta-analysis. Glob Change Biol 25: 3018-3030. https://doi.org/10.1111/gcb.14705

\section{Figures}


(A)

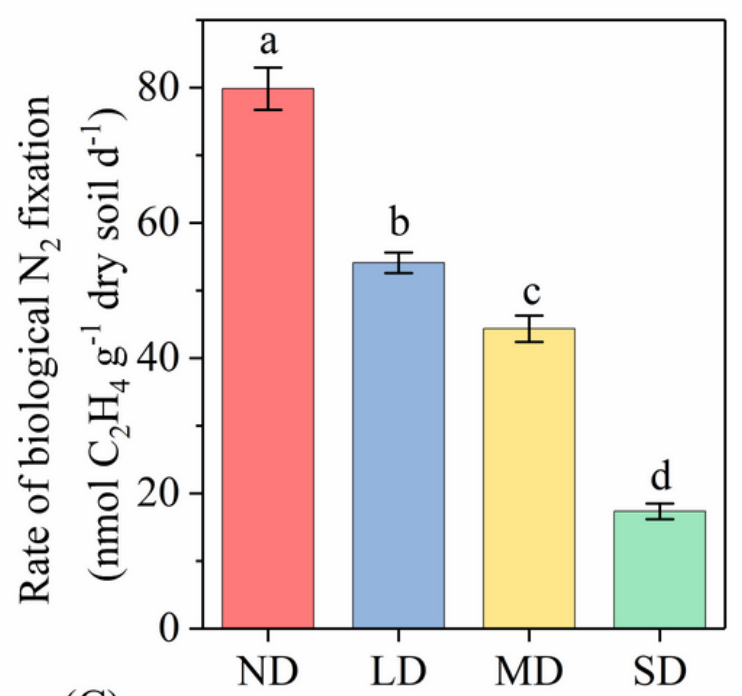

(C)

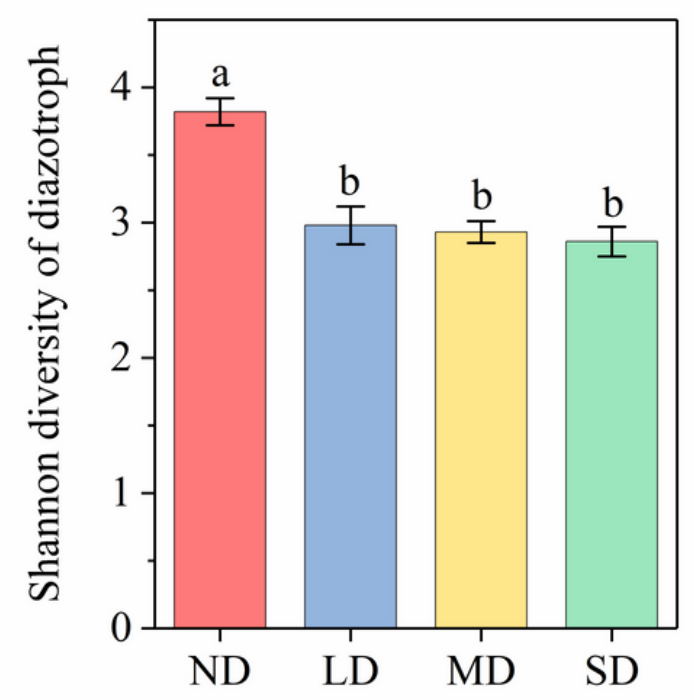

(B)

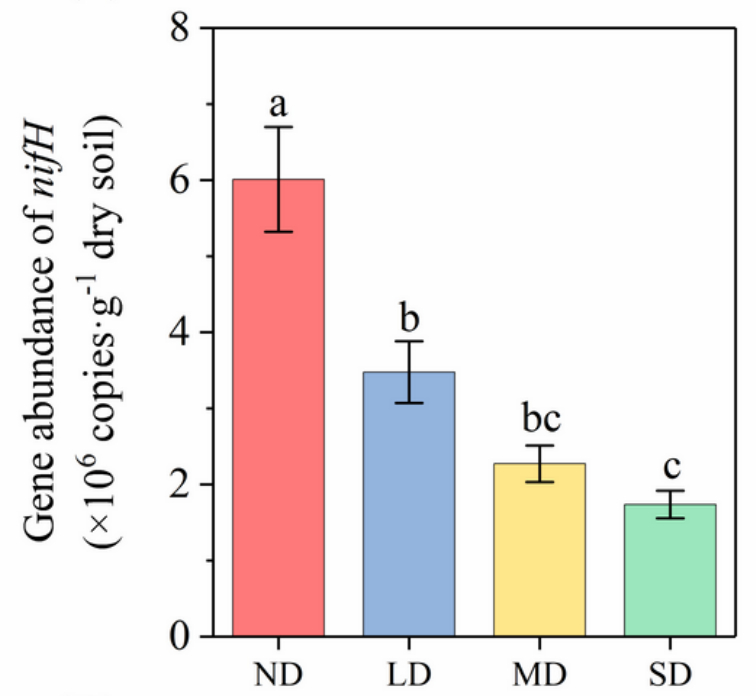

(D)

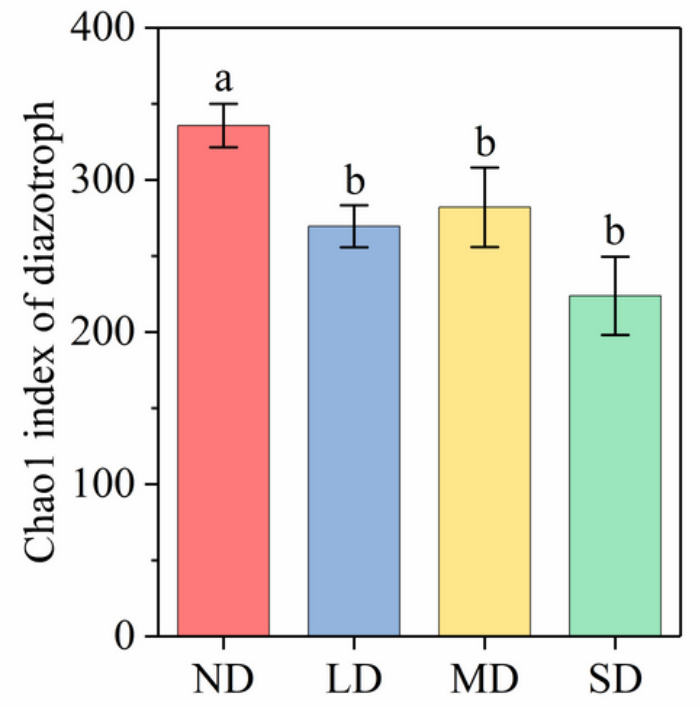

Figure 1

The rate of biological N2 fixation (A), nifH gene abundance (B), the Shannon Index of diazotrophs (C) and the Chao1 Index of diazotrophs (D) for each meadow. ND: non-degraded meadow; LD: lightly degraded meadow; MD: moderately degraded meadow; SD: severely degraded meadow. Error bars indicate standard errors of the mean $(n=5)$. Values above the bars represent mean \pm SE for each of the four meadow types. Different letters mean significant differences under different treatments (Duncan's test, $\mathrm{P}<0.05$ ). 
(A)

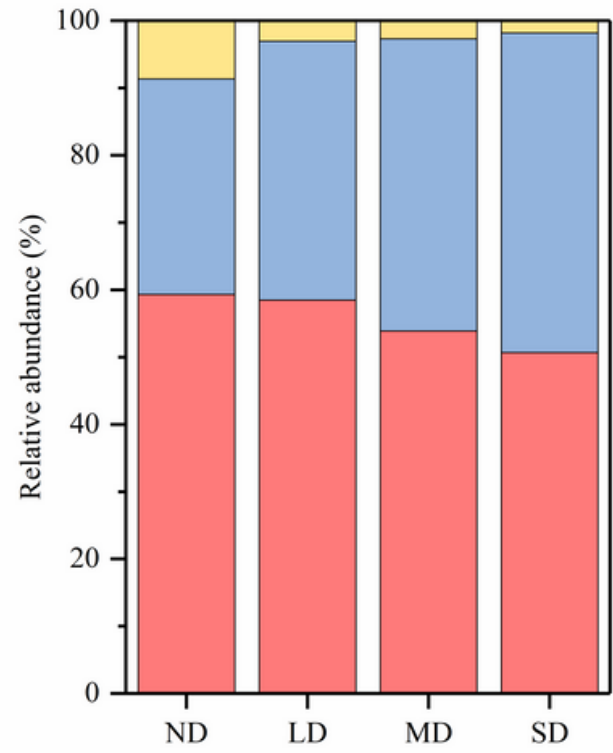

(B)

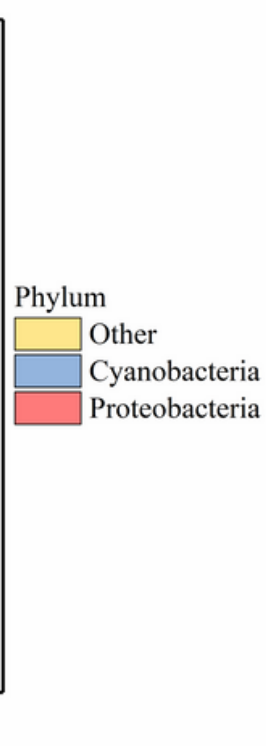

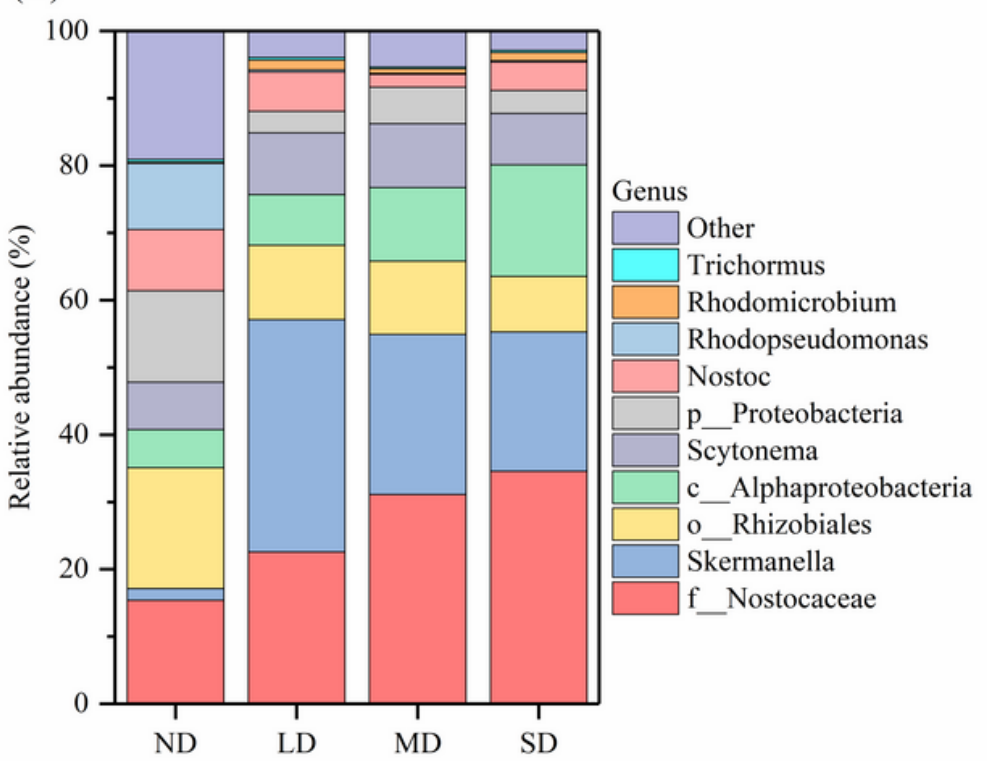

Figure 2

Relative abundance of diazotrophs at the phylum level (A) and genus level (B) for each meadow. ND: non-degraded meadow; LD: lightly degraded meadow; MD: moderately degraded meadow; SD: severely degraded meadow.

(A)

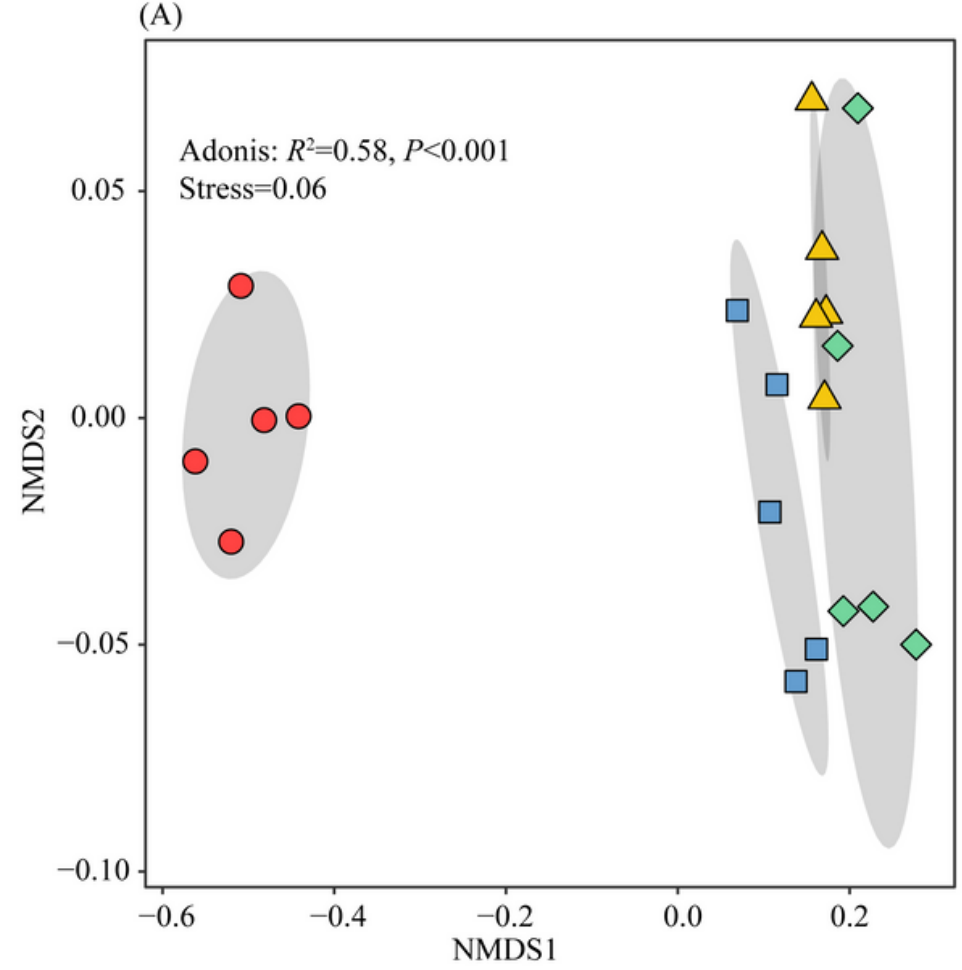

(B)

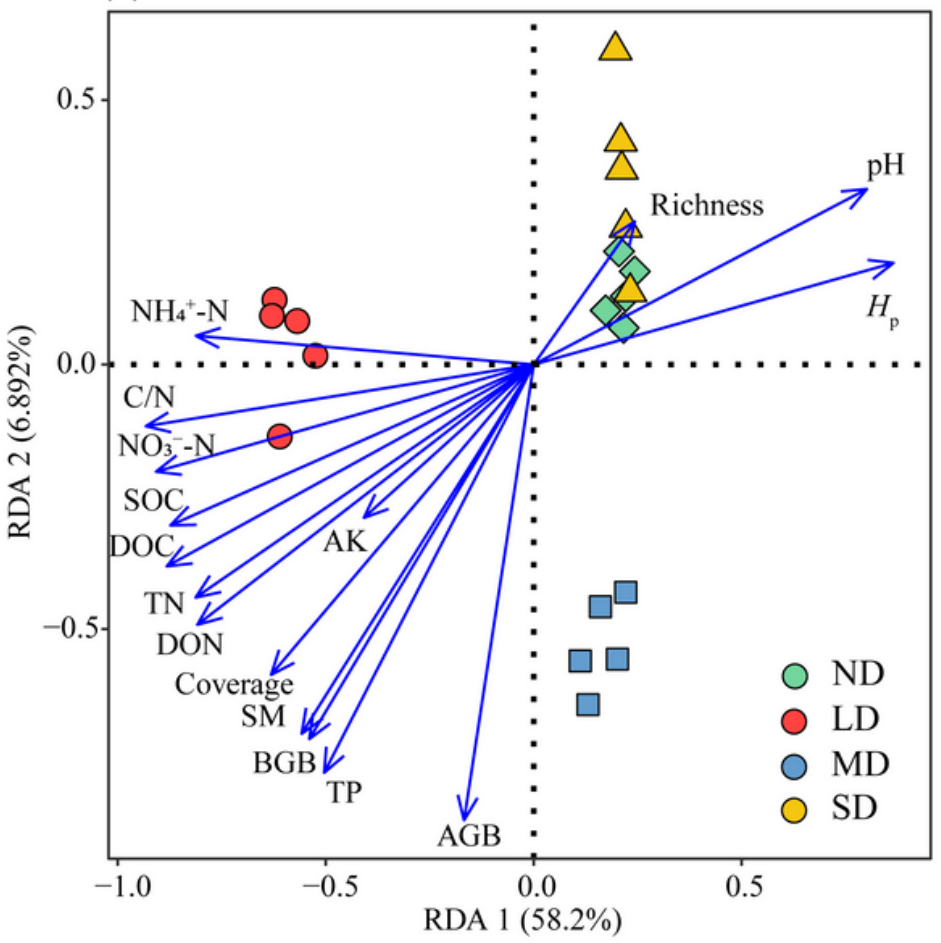

Figure 3

Nonmetric multidimensional scaling (NMDS) plots of diazotrophs at the OTU level (A), and Redundancy analysis (RDA) based on OUT data with vegetation and soil properties in four meadows. ND: non-degraded meadow; LD: lightly degraded meadow; MD: moderately degraded meadow; SD: severely degraded meadow. AP: available P; DOC: dissolved organic C; 
DON: dissolved organic N; SOC: soil organic C; TN: total N; TP: total P; AK: available K; SM: soil moisture; C/N: the ratio of SOC and TN; AGB: plant aboveground biomass; BGB: plant belowground biomass; Hp: plant Shannon diversity index.

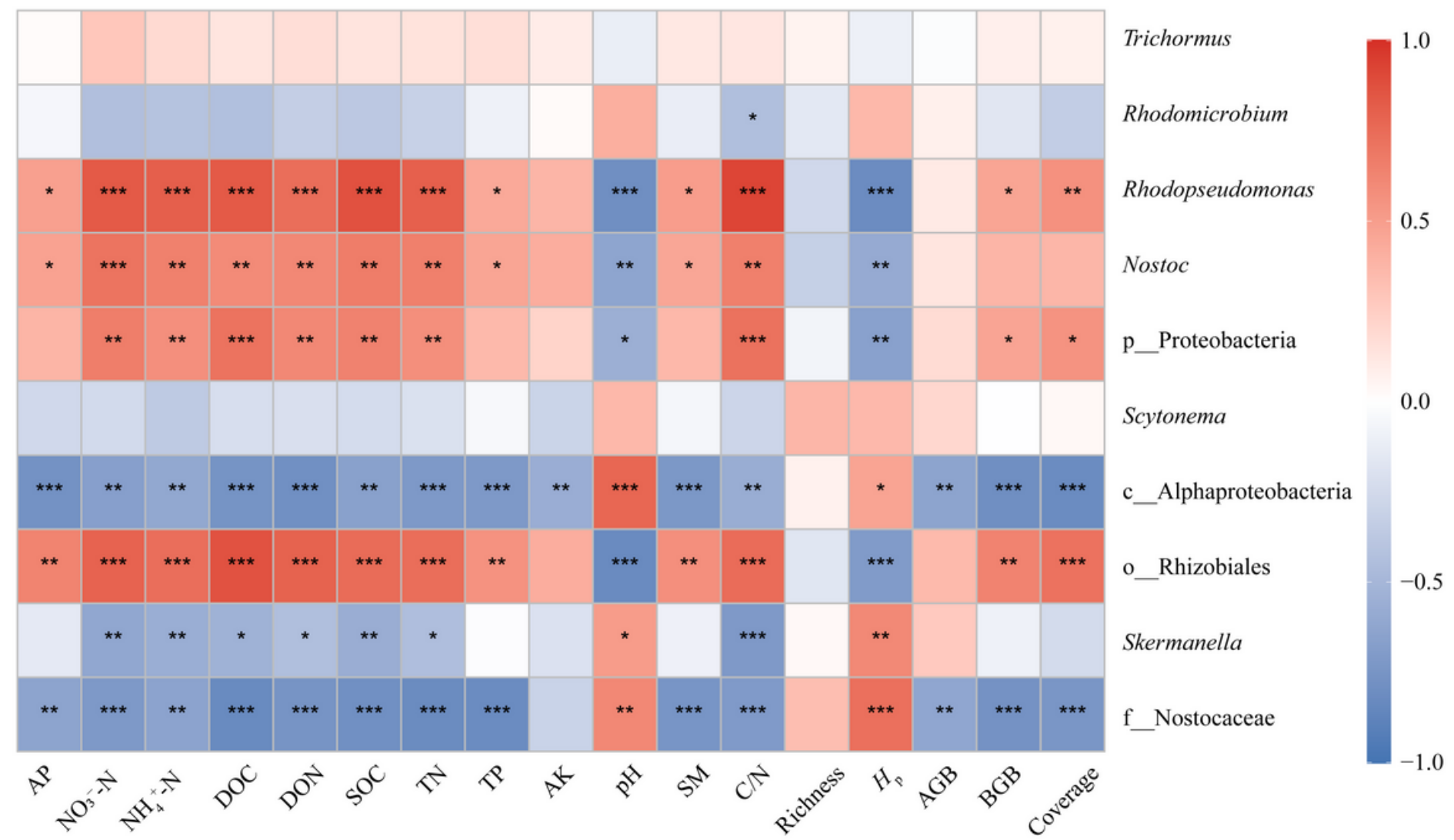

\section{Figure 4}

Heatmap for Pearson's correlation between vegetation and soil properties and the relative abundance of dominant diazotrophs at the genus level. AP: available P; DOC: dissolved organic C; DON: dissolved organic N; SOC: soil organic C; TN: total N; TP: total P; AK: available K; SM: soil moisture; C/N: the ratio of SOC and TN; AGB: plant aboveground biomass; BGB: plant belowground biomass; Hp: plant Shannon diversity. 


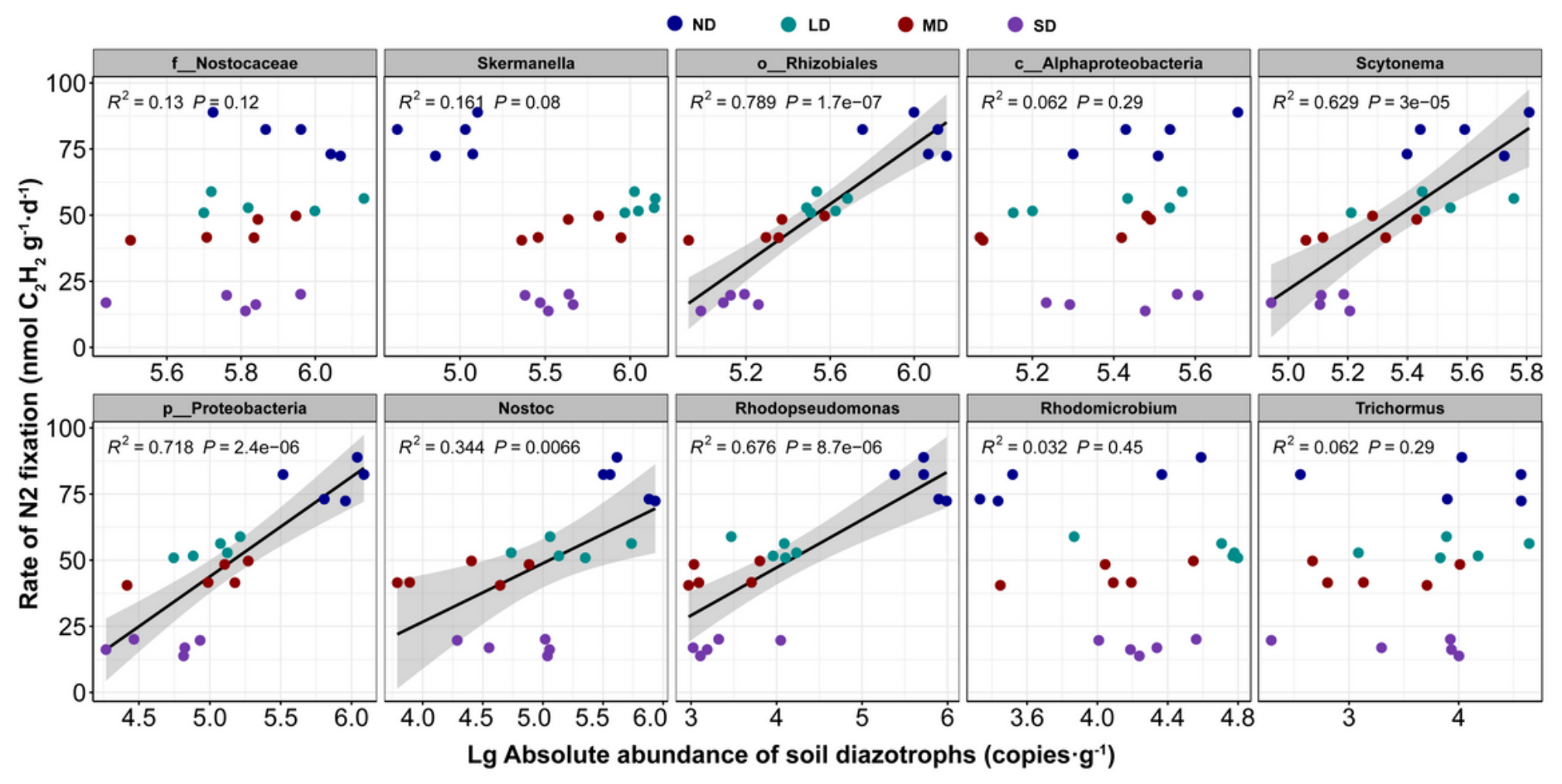

Figure 5

Ordinary least-squares (OLSRM) regression shows the relationships between the rate of biological N2 fixation and the absolute abundance of dominant diazotrophic genera. The gray shaded area shows the $95 \%$ confidence interval of the fit. ND: non-degraded meadow; LD: lightly degraded meadow; MD: moderately degraded meadow; SD: severely degraded meadow. 
(A)

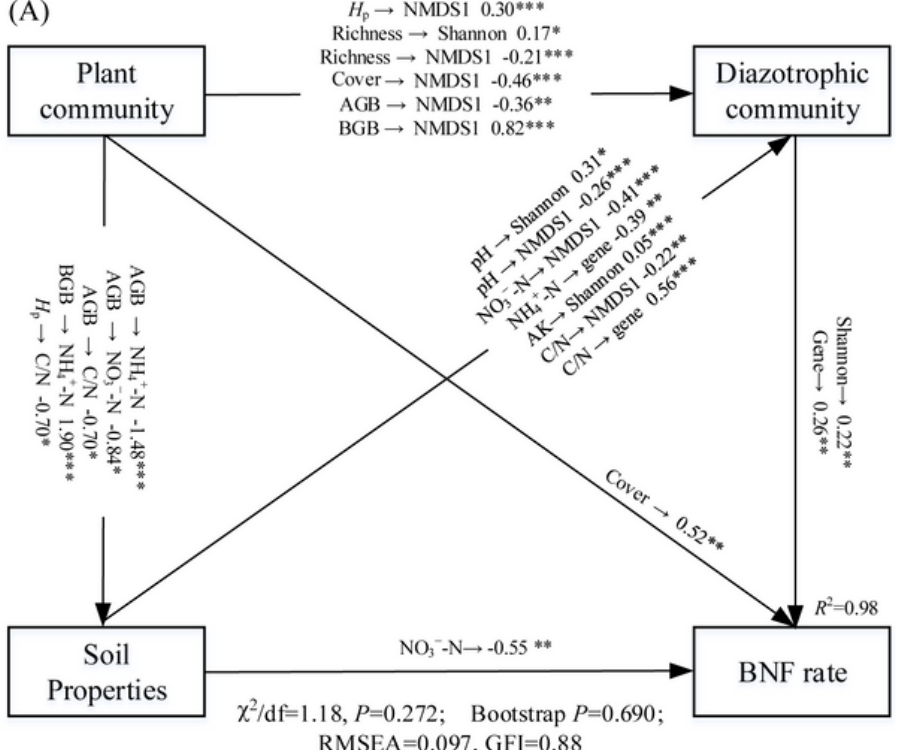

RMSEA $=0.097, \mathrm{GFI}=0.88$

(C)

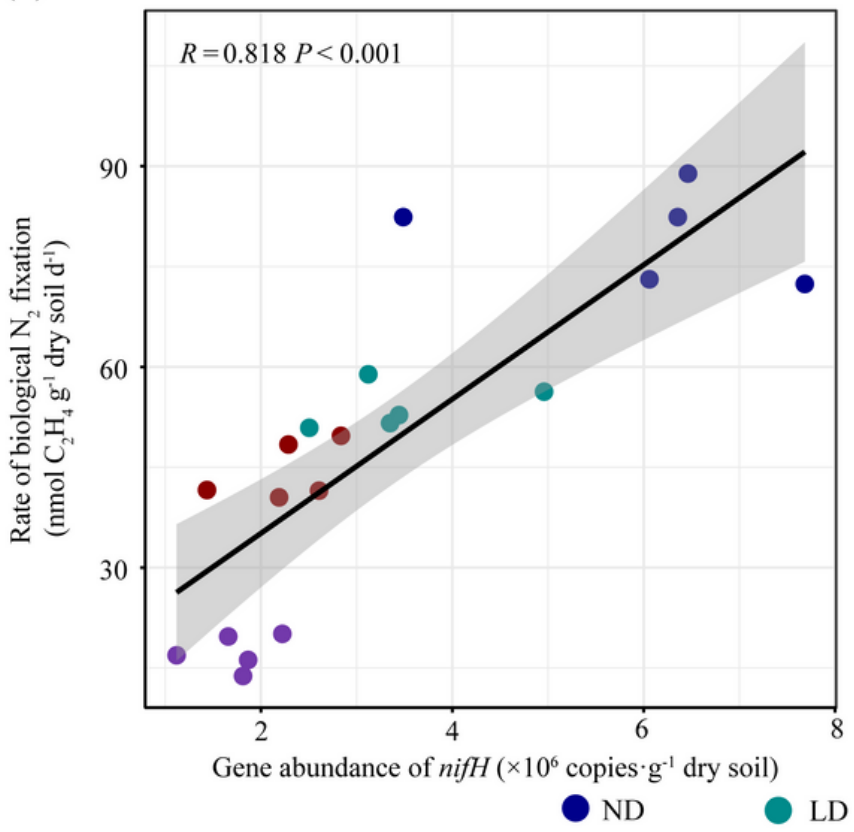

(B)

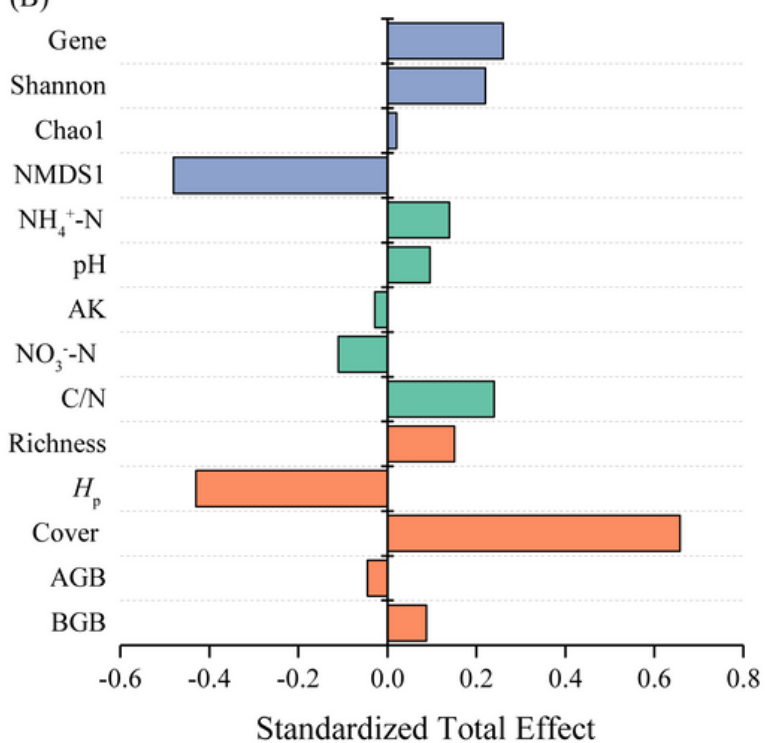

(D)

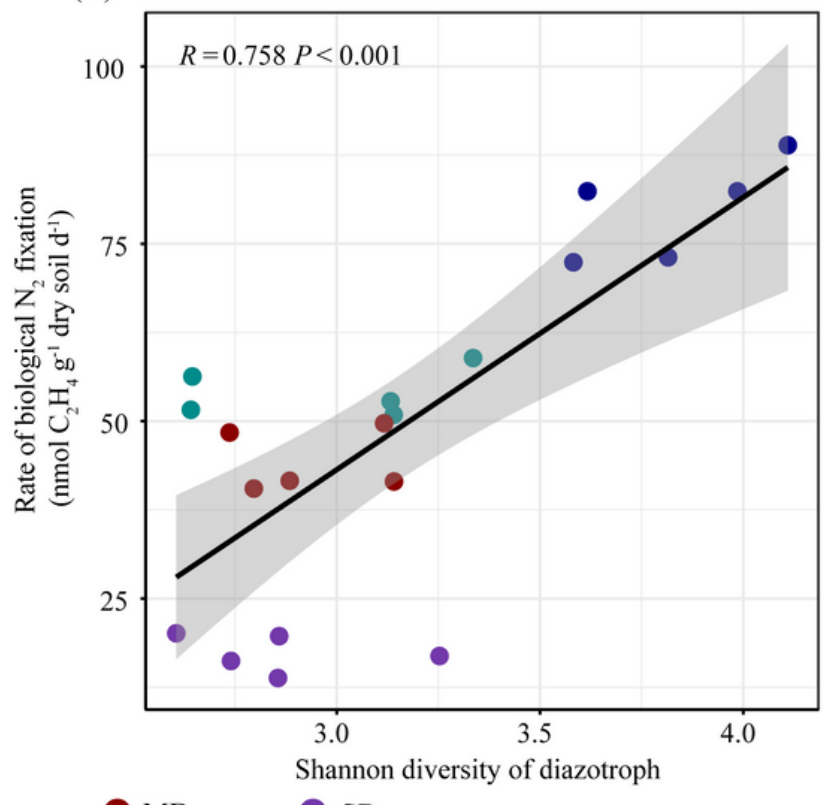

MD SD

\section{Figure 6}

Structural equation modeling (SEM) showing the effect of soil variables ( $\mathrm{NH} 4+, \mathrm{NO}--\mathrm{N}, \mathrm{AK}, \mathrm{pH}, \mathrm{C} / \mathrm{N})$, plant characteristics (AGB, BGB, Cover, Hp, Richness), and community characteristics of diazotrophs (Shannon and Chao1 diversity indexes, gene abundance and NMDS1) on the rate of biological N2 fixation in alpine meadow (A), the standardized total effects from the SEM (B), and ordinary least-squares (OLSRM) regression model shows the relationships between the rate of biological N2 fixation, gene abundance of nifH (C) and Shannon diversity of diazotroph (D). The gray shaded area shows the $95 \%$ confidence interval of the fit. ND: non-degraded meadow; LD: lightly degraded meadow; MD: moderately degraded meadow; SD: severely degraded meadow. AK: available K; SM: soil moisture; C/N: the ratio of SOC and TN; AGB: plant aboveground biomass; BGB: plant belowground biomass; Hp: plant Shannon diversity index; Richness: plant richness index; Cover: vegetation cover; Gene: gene abundance of nifH; Shannon: Shannon diversity index of diazotrophs; Chao1: Chao1 diversity index of diazotrophs; NMDS1: the first component from the NMDS); BNF rate: the rate of biological N2 fixation. The components within plant community, soil properties and diazotrophic community are included in the model 
as independent observed variables, but in this figure are grouped for simplicity. Numbers within the arrows show standardized path coefficients and indicate the effect size of the relationship among variables. Only significant relationships are shown ( $\left.{ }^{*} \mathrm{P}<0.05 ;{ }^{*} \mathrm{P}<0.01 ; * \star * \mathrm{P}<0.001\right)$ in SEM. The goodness-of-fit of the model is shown at the bottom of the figure. RMSEA: root mean square error of approximation; GFI: goodness of fit index.

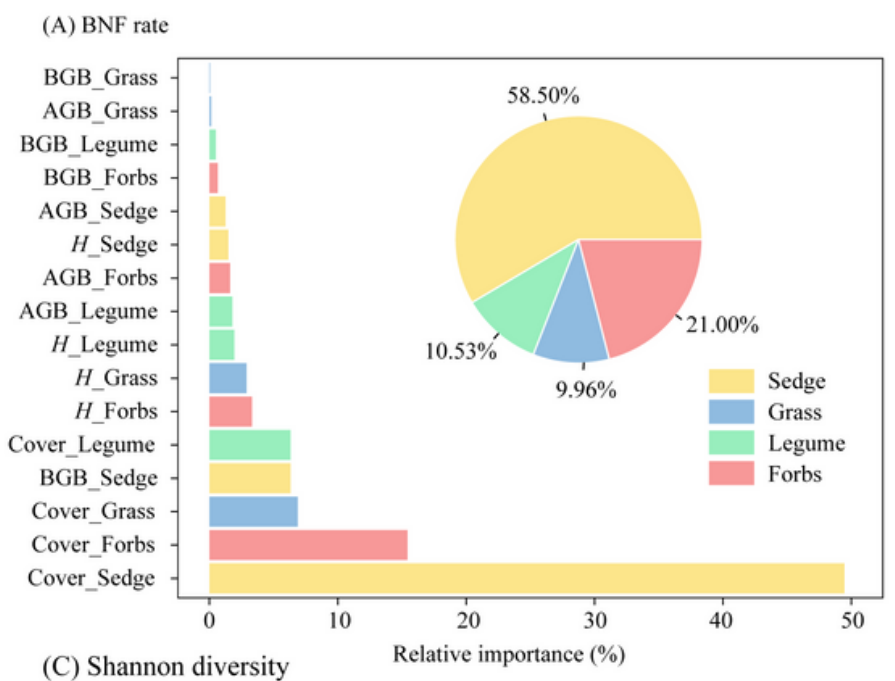

(B) Gene abundance
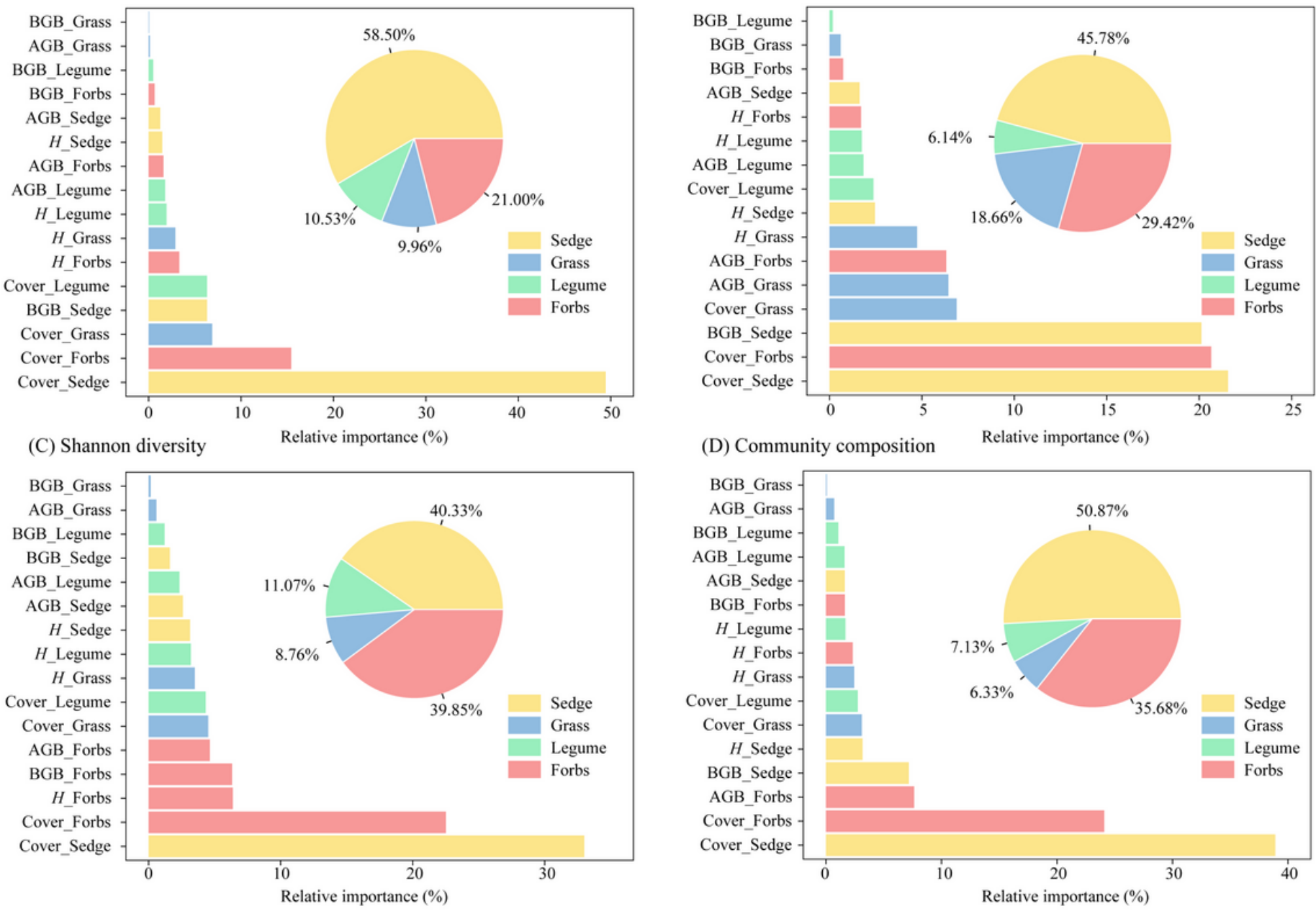

(D) Community composition

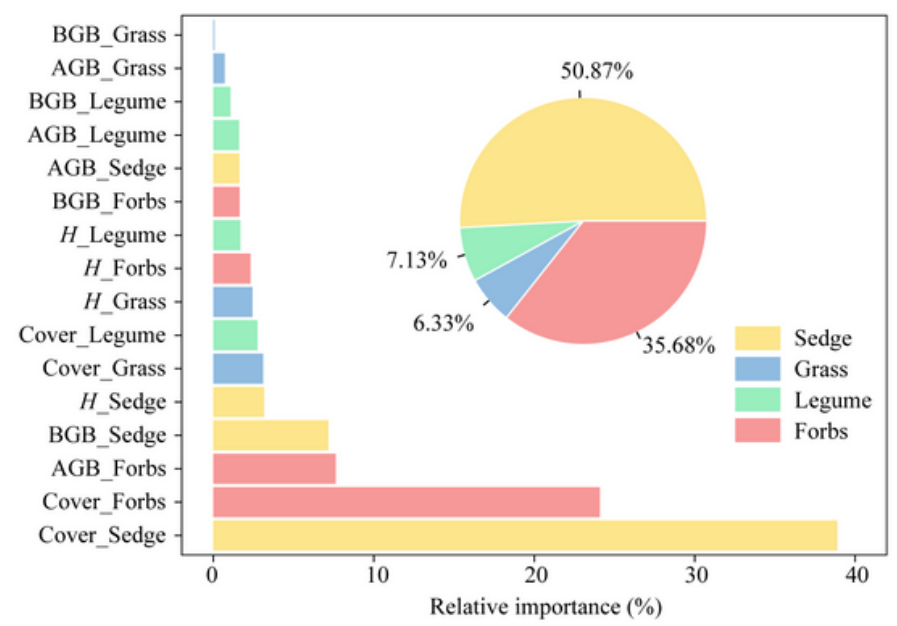

\section{Figure 7}

Aggregated boosted tree (ABT) analysis revealed the effect of plant functional groups on (A) BNF rate (B) abundance of the nifH gene (C) a-diversity of diazotrophs (represented by Shannon index) and (D) community composition of soil diazotrophs (represented by the first component from the NMDS). AGB: plant aboveground biomass; BGB: plant belowground biomass; $\mathrm{H}$ : plant Shannon diversity index; Cover: vegetation cover.

\section{Supplementary Files}

This is a list of supplementary files associated with this preprint. Click to download.

- AppendixA.Supplementarydata.docx 